\title{
Characterization of Dmrt3-Derived Neurons Suggest a Role within Locomotor Circuits
}

\author{
Sharn Perry, ${ }^{\star}$ Martin Larhammar, ${ }^{\star}$ Jennifer Vieillard, Chetan Nagaraja, ${ }^{-M a r k u s ~ M . ~ H i l s c h e r, ~} @$ Atieh Tafreshiha, \\ Fadi Rofo, ${ }^{\circ}$ Fabio V. Caixeta, and $®$ Klas Kullander \\ Department of Neuroscience, Uppsala University, 75124 Uppsala, Sweden
}

\begin{abstract}
Neuronal networks within the spinal cord, collectively known as the central pattern generator (CPG), coordinate rhythmic movements underlying locomotion. The transcription factor doublesex and mab-3-related transcription factor 3 (DMRT3) is involved in the differentiation of the dorsal interneuron 6 class of spinal cord interneurons. In horses, a non-sense mutation in the Dmrt3 gene has major effects on gaiting ability, whereas mice lacking the Dmrt3 gene display impaired locomotor activity. Although the Dmrt3 gene is necessary for normal spinal network formation and function in mice, a direct role for Dmrt3-derived neurons in locomotor-related activities has not been demonstrated. Here we present the characteristics of the Dmrt3-derived spinal cord interneurons. Using transgenic mice of both sexes, we characterized interneurons labeled by their expression of Cre driven by the endogenous Dmrt3 promoter. We used molecular, retrograde tracing and electrophysiological techniques to examine the anatomical, morphological, and electrical properties of the Dmrt3Cre neurons. We demonstrate that inhibitory Dmrt3-Cre neurons receive extensive synaptic inputs, innervate surrounding CPG neurons, intrinsically regulate CPG neuron's electrical activity, and are rhythmically active during fictive locomotion, bursting at frequencies independent to the ventral root output. The present study provides novel insights on the character of spinal Dmrt3-derived neurons, data demonstrating that these neurons participate in locomotor coordination.
\end{abstract}

Key words: central pattern generator; $\mathrm{CPG}$; Dmrt3; interneuron; locomotion

Significance Statement

In this work, we provide evidence for a role of the Dmrt3 interneurons in spinal cord locomotor circuits as well as molecular and functional insights on the cellular and microcircuit level of the Dmrt3-expressing neurons in the spinal cord. Dmrt3 neurons provide the first example of an interneuron population displaying different oscillation frequencies. This study presents novel findings on an under-reported population of spinal cord neurons, which will aid in deciphering the locomotor network and will facilitate the design and development of therapeutics for spinal cord injury and motor disorders.

\section{Introduction}

Locomotion is coordinated by neuronal networks in the spinal cord known as central pattern generators (CPGs), which provide

Received Jan. 31, 2018; revised Nov. 27, 2018; accepted Dec. 10, 2018.

Author contributions: S.P., M.L., J.V., C.N., M.M.H., F.V.C., and K.K. edited the paper; M.L. wrote the first draft of the paper; S.P., M.L., C.N., M.M.H., F.V.C., and K.K. designed research; S.P., M.L., J.V., C.N., M.M.H., A.T., F.R., and F.V.C. performed research; K.K. contributed unpublished reagents/analytic tools; S.P., M.L., J.V., C.N., M.M.H., F.V.C., and K.K. analyzed data; S.P., M.L., F.V.C., and K.K. wrote the paper.

This work was financed by Grants from the Swedish Medical Research Council, Hållsten, Ländells, Swedish Brain Foundations, the Swedish Foundation for Cooperation in Research and Higher Education, and CNPq-Brazil; by a scholarship from CNPq-Brazil to F.V.C.; and by the Foundation for International Cooperation in Research and Higher Education and the Brazilian Federal Agency for Support and Evaluation of Graduate Education-CAPES to M.M.H. We thank George Nascimento, Richardson Leao, Evangelos Grivogiannis, Ockie Bernadotte, and Qun Zhang for technical assistance; Henrik Boije for valuable input on the paper; and Biological Visualisation Uppsala for imaging support.

The authors declare no competing financial interests.

*S.P. and M.L. contributed equally to this work.

Correspondence should be addressed to Klas Kullander at klas.kullander@neuro.uu.se or Martin Larhammar at martin.larhammar@gmail.com. a rhythmic and patterned output to motor neurons (Grillner, 1975; Kullander, 2005; Goulding, 2009). The neuronal classes involved in spinal locomotor CPG networks arise from ventral progenitor domains (V0-V3) and the dorsal interneuron 6 (dI6) progenitor domain (Goulding, 2009; Dyck et al., 2012) established during spinal cord development. Although the dI6 population has been suggested to take part in the coordination of left-right alternation (Goulding, 2009; Rabe et al., 2009; Dyck et al., 2012), it remains one of the least characterized spinal interneuron classes. Electrophysiological recordings revealed a dorsal subset of putative dI6 neurons to be rhythmically active during fictive locomotion, some of which might be involved in

S. Perry's present address: Wicking Dementia Research and Education Center, University of Tasmania, Hobart, TAS 7000, Australia.

M. Larhammar's present address: Denali Therapeutics, South San Francisco, CA 94080.

https://doi.org/10.1523/JNEUROSCI.0326-18.2018

Copyright $\odot 2019$ the authors $\quad 0270-6474 / 19 / 391771-12 \$ 15.00 / 0$ 
locomotor rhythm generation whereas others may participate in locomotor patterning (Dyck et al., 2012). The doublesex and mab-3 related transcription factor 3 (DMRT3) is exclusively expressed in dI6 interneurons and displays a partial overlap with another transcription factor expressed in dI6 neurons, Wilms Tumor 1 (WT1; Goulding, 2009; Andersson et al., 2012). Within the dI6 neural assembly, the Dmrt3-derived subpopulation is comprised of ipsilateral and contralateral inhibitory neurons that innervate motor neurons (Andersson et al., 2012). Because the dI6 subpopulation expressing WT1 expands in the absence of Dmrt3 (Andersson et al., 2012), it is currently unclear whether Dmrt3-derived neurons themselves participate in locomotor coordination.

A non-sense mutation in the horse DMRT3 gene, which leads to a premature stop codon, was recently found to be permissive for horses' ability to perform alternate gaits in addition to the three natural gaits: walk, trot and gallop (Andersson et al., 2012). The "Gait-keeper" mutation in horse DMRT3 has been under strong selection by humans worldwide, due to its impact on locomotor performance and the improved resistance to the trotgallop switch at higher speeds. In the same study, a Dmrt3 gene deletion in mice affected dI6 neuron development with consequences for the CPG output. Neonatal Dmrt3-null mice showed severe problems coordinating hindlimb movements, whereas adult Dmrt3-null mice displayed gait abnormalities and difficulties running at high speeds (Andersson et al., 2012). Thus, the Dmrt3 gene deletion results in faulty development of the dI6 subdomain, resulting a disorganized locomotor network. In the present study, we generated a Dmrt3-Cre mouse line to investigate the character of spinal Dmrt3 interneurons using functional imaging, anatomical and electrophysiological techniques.

\section{Materials and Methods}

Mice. All animal procedures were approved by the local Swedish ethical committee (permits C248/11 and C135/14). All animals were maintained on a mixed C57BL/6 background and only heterozygous Dmrt3 ${ }^{\mathrm{Cre}}$ $(\mathrm{Cre}+)$ mice were used to avoid the potential risk of abnormal Dmrt3 gene expression. Mice $>6$ weeks of age are referred to as adults.

Generation of Dmrt $3^{\mathrm{Cre}}$ mice. Dmrt $3^{\mathrm{Cre}}$ knock-in animals were generated using the Zinc Finger Nuclease (ZFN) technology (Sigma-Aldrich). ZFNs mRNA and a donor DNA vector containing improved cyclic recombinase (iCre) followed by a $2 \mathrm{~A}$ peptide sequence positioned between homology arms were introduced to B6D2F1 zygotes. The 2A peptide coding sequence (Trichas et al., 2008) allows for cotranslation of the Dmrt3 promoter-driven iCre followed by Dmrt3 (similar to internal ribosomal entry site). ZFN binding sites (upper case) and cutting site (lower case, underlined) in Dmrt3 exon 1: 5'-GGCTACGGCT CCCCCtacctgTACATGGGCGGCCCGGTG. Founders were verified for iCre by PCR using the following primers: 5 '-ACGAGTGATGAGGTT CGCAAGA (forward) and 5'-ACCGACGATGAAGCATGTTTAG (reverse). The $\mathrm{Dmrt3} 3^{\mathrm{Cre}}$ expression pattern was validated after breeding with Gt(ROSA)26Sort ${ }^{\text {m14(CAG-tdTomato)Hze }}$ reporter mice, followed by Dmrt3 in situ hybridization (ISH) and Dmrt3 immunohistochemistry. Additional mouse lines used in combination with Dmrt3 ${ }^{\mathrm{Cre}}$ :

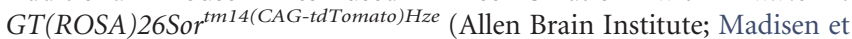
al., 2010).

Molecular analysis. ISH and immunohistochemistry (IHC) were conducted as previously described (Enjin et al., 2010; Andersson et al., 2012). Briefly, ISH experiments were performed with an antisense DIG-labeled mRNA probe for Dmrt3 (nucleotide 1404-2401, NM_177360.3), Vglut2 (nucleotides 1616-2203, NM_080853.2), and Viaat (nucleotides 5882072, NM_008744.2).

IHC was conducted on $4 \%$ formalin fixed $12-16 \mu \mathrm{m}$ thin spinal cord cryosections and $40 \mu \mathrm{m}$ vibratome sections. Primary antibodies were incubated in $5 \%$ goat serum, $0.3 \%$ BSA in PBS or Blocking reagent (Roche) for $24-48 \mathrm{~h}$ at $4^{\circ} \mathrm{C}$. The following antibodies were used; poly- clonal rabbit anti-calbindin (1:5000; CB38, Swant), polyclonal goat antiCHAT (1:100; AB144P, Millipore), anti-Dmrt3 guinea pig sera (Innovagen), anti-Glycine rat polyclonal (1:5000; IG1002, Immunosolution), polyclonal guinea pig anti-Vacht (1:600; Millipore), polyclonal guinea pig anti-Vglut1 (1:500; gift from Dr. Kaneko, Kyoto University, Japan), polyclonal guinea pig anti-Vglut2 (1:10000; AB2251, Millipore), polyclonal rabbit anti-Vmat2 (1:500; 138302, Synaptic Systems), anti-dsRed (1:400; 632498, BioNordika), polyclonal rabbit anti-Slc10a4 (1:800; HPA028835, Sigma-Aldrich), polyclonal rabbit anti-Viaat (1:500; gift from Bruno Gasnier, Université Paris Descartes, CNRS, Paris; Dumoulin et al., 1999), polyclonal rabbit anti-Dbx1 (1:10000; gift from Thomas Jessell, Columbia University, New York), polyclonal guinea pig antiLbx1 (1:2000; gift from Carmen Birchmeier, Max Delbrück Center, Berlin), monoclonal mouse anti-Synaptophysin (1:500; S-5768, SigmaAldrich) and monoclonal mouse anti-WT1 (1:1; 6F-H2, Dako). Antigen retrieval using citrate buffer was performed before immunolabeling with WT1 antibody. Secondary antibodies were incubated in PBS with DAPI; Alexa donkey anti-rabbit 488 and 647, anti-guinea pig 488, and 647, anti-goat 488, anti-mouse 647 (Invitrogen), and DyLight goat antimouse 488 (Abcam).

Retrograde tracing. To examine the projections of Dmrt3-Cre neurons, tracing experiments were conducted on six Dmrt3;tdT-positive pups aged P0/P1 as previously described (Rabe et al., 2009; Andersson et al., 2012). Briefly, two horizontal cuts (for intersegmental tracing targeting ascending/descending/bifurcating neurons) were made in Dmrt3 ${ }^{\text {Cre }}$; $t d T$ neonatal mouse spinal cords at L1 and between L3 and L4. Fluoresceindextran-amine (FDA; $3000 \mathrm{MW}$ ) and Alexa Fluor 647 (10,000 MW; Invitrogen) tracers were applied and spinal cords were incubated overnight at room temperature. Traced spinal cords were fixed in $4 \%$ formaldehyde for 1 week before transverse sectioning $(60 \mu \mathrm{m})$ on a vibratome (Leica VT 1000s). Images were acquired on a fluorescence (OlympusBX61WI) or confocal microscope (Zeiss LSM 510 META) and processed using ImageJ (http://imagej.nih.gov/ij/). Venn diagrams were generated using eulerAPE (Micallef and Rodgers, 2014).

Single-cell PCR. Brains from Dmrt ${ }^{\text {Cre }}$; $t$ TT adult animals were removed and sliced $(300 \mu \mathrm{m})$ on a vibratome (Leica VT 1200). Dmrt3 ${ }^{\mathrm{Cre}}$; $t d T$ cells identified by RFP expression in the motor cortex (M1 and M2), were individually picked using pipettes pulled from borosilicate glass pipettes $($ GC150F-10, 1.5 outer diameter $\times 0.86$ inner diameter $\times 100 \mathrm{~L}$ $\mathrm{mm}$, Harvard Apparatus) on a PC-10 gravitational pipette puller (Narishige).

The content of each pipette was transferred immediately into individual pre-chilled $200 \mu \mathrm{l}$ tubes containing $6 \mu \mathrm{l}$ of a freshly prepared solution of $20 \mathrm{U}$ of RNaseOUT (Fermentas) and $8.3 \mathrm{~mm}$ DTT (Invitrogen). Samples were snap frozen on dry ice until use. Frozen samples were thawed on ice and subjected to cDNA synthesis for $1 \mathrm{~h}$ using $0.5 \mathrm{~mm}$ dNTPs mix, $1.25 \mu \mathrm{M}$ random hexamers (Invitrogen), $40 \mathrm{U}$ of RNase inhibitor (Fermentas), $100 \mathrm{U}$ of M-MLV RT (Invitrogen), $50 \mathrm{~mm}$ Tris- $\mathrm{HCl}, 75 \mathrm{~mm} \mathrm{KCl}$, and $3 \mathrm{~mm} \mathrm{MgCl}_{2}, \mathrm{pH}$ 8.3. The RT enzyme was denatured and the cDNAs stored at $-80^{\circ} \mathrm{C}$ until further use. A first round PCR was performed using $1.5 \mathrm{~mm} \mathrm{MgCl}_{2}, 10 \mathrm{pmol}$ of each primer, $1.0 \mathrm{U}$ of Maxima Hot Start Taq Polymerase (Fermentas), $20 \mathrm{~mm}$ Tris- $\mathrm{HCl}$, and $10 \mathrm{~mm} \mathrm{KCl} \mathrm{pH} 8.3$ for 35 cycles with an annealing temperature of $55^{\circ} \mathrm{C}$. A second round of PCR was performed using $10 \%$ of the first PCR. Primers were designed based upon sequences deposited in the GenBank database (http:// www.ncbi.nlm.nih.gov/nucleotide). They were designed to bind different exons, therefore they detect only mRNA and not genomic DNA. Ladders: $1 \mathrm{~kb}$ plus and $100 \mathrm{bp}$ (Fermentas). Right primers are followed by left primers:

GAD 67: 5' -ccaatagcctggaagagaagag- $3^{\prime}$ and $5^{\prime}$-tcccatcaccatctttatttga-3';

GAD 67 nested: $5^{\prime}$-ccaatagcctggaagagaagag- $3^{\prime}$ and $5^{\prime}$-tcccatcaccatctttatttga-3';

$\beta$-actin $5^{\prime}$-ctcttttccagccttccttctt- $3^{\prime}$ and $5^{\prime}$-agtaatctccttctgcatcctgtc- $3^{\prime}$.

$R T-q P C R$. The entire spinal cord of the embryonic stages and the lumbar segment of the postnatal stages were dissected in ice-cold PBS and snap frozen in liquid nitrogen. RNA extraction was made using the RNeasy Mini Kit (Qiagen). The reverse transcription was performed using the Quantitect Reverse Transcription Kit (Qiagen). The qPCR was conducted using the QuantiFast SYBR Green PCR Kit (Qiagen). Tripli- 
cates were performed on two different biological samples from each stage. The PCR was run in the CFX96 Touch Real-Time PCR Detection System (Bio-Rad). Relative transcript levels of Dmrt3 was measured in triplicates (primer sequences: AGCGCAGCTTGCTAAACC, GAGCTCCTCTGATCGGTGTC) and normalized to ActB (primer sequences: CACTGTCGAGTCGCGTCC, AGCGATATCGTCCGCATCCA) and Tbp (primer sequences: CCTTGTACCCTTCACCAATGAC, ACAGCCAAGATTCACGGTAGA), using the comparative $C t(\Delta \Delta C t)$ method.

Electrophysiology. Whole-cell patch-clamp electrophysiology recordings from transverse, lumbar spinal cord slices of Dmrt $3^{\mathrm{Cre}}$; $t d \mathrm{~T}$ neonates (thickness: $300 \mu \mathrm{m}$ ) were done as previously described (Lamotte d'Incamps and Ascher, 2008; Perry et al., 2015). One or at the most two cells were used per slice. In brief, slices were incubated in equilibrated $\left(95 \% \mathrm{O}_{2}\right.$ and $5 \% \mathrm{CO}_{2}$ ) artificial CSF (aCSF) containing the following in (mM): $130 \mathrm{NaCl}, 2.5 \mathrm{KCl}, 1.25 \mathrm{NaH}_{2} \mathrm{PO}_{4}, 26 \mathrm{NaHCO}_{3}, 2 \mathrm{CaCl}_{2}$, and 1 $\mathrm{MgCl}_{2}$ for $45 \mathrm{~min}$ and subsequently held at room temperature $\left(22-25^{\circ} \mathrm{C}\right)$ throughout experimental procedures. Dmrt ${ }^{\mathrm{Cre}}$; $t d \mathrm{~T}$ spinal cords for whole-cord preparations were dissected as previously described (Andersson et al., 2012) and allowed to equilibrate in oxygenated aCSF for 30 min before recordings. Dmrt ${ }^{\mathrm{Cre}}$; $t d \mathrm{~T}$ cells were visualized with a BX51WI Olympus microscope fitted with infrared differential interference contrast optics and a Lambda LS Xenon Arc lamp (Sutter Instruments) for fluorescent light. Patch electrodes (5-9 M $\Omega$ ) from borosilicate glass capillaries (GC150F-10, Harvard Apparatus) pulled on a PC-10 gravitational pipette puller (Narishige) contained a $\mathrm{K}^{+}$-based internal solution (in mM): $130 \mathrm{~K}$-gluconate, $7 \mathrm{NaCl}, 10 \mathrm{HEPES}, 0.1 \mathrm{EGTA}, 0.3 \mathrm{MgCl}_{2}$, and 2 ATP 0.5 GTP, with $\mathrm{pH}$ adjusted to 7.2 using $\mathrm{KOH}$ with an osmolarity between 280-300 mOsm/L. Biocytin (1.5-2 mg; Sigma-Aldrich) was routinely dissolved in the internal solution. The liquid junction potential was calculated as $14.4 \mathrm{mV}$ using Clampex software (10.2). Dorsal roots were occasionally mounted into glass suction electrodes and stimulated at $1.5 \times$ threshold (A360 WPI Stimulus isolator, World Precision Instruments). Patch-clamp recordings were made using a MultiClamp 700B amplifier (Molecular Devices) and digitalized with a BNC block (BNC2110 National Instruments), low-pass filtered at $4 \mathrm{kHz}$ and digitized at 10 $\mathrm{kHz}$, and acquired/analyzed in WinWCP software (Dr. J. Dempster, University of Strathclyde, Glasgow, UK), AxoGraph X and/or MATLAB (MathWorks).

Firing properties were examined by current injections ( -200 to 150 $\mathrm{pA}, 1 \mathrm{~s}, 50 \mathrm{pA}$ increments; $0-100 \mathrm{pA}, 1 \mathrm{~s}, 10 \mathrm{pA}$ increments) with a $3 \mathrm{~s} \mathrm{step}$ interval to assure membrane potentials returned to baseline. The spike rate was calculated as the number of spikes in a $1000 \mathrm{~ms}$ duration pulse. In frequency (f) versus current $(I)$ plots, frequency was computed over the last $500 \mathrm{~ms}$ of traces from trains of action potentials (APs) evoked by $1000 \mathrm{~ms}$ duration pulses (10 and $50 \mathrm{pA}$ increments). In spike-frequency adaptation plots, the initial frequency was defined as the inverse of the first interspike interval during a 50/100/150 pA current step. The steadystate (SS) frequency was defined as the average of the inverse of the last three interspike intervals in a 50/100/150 pA current step. The adaptation ratio was calculated as the inverse of the mean of the last three interspike intervals during a 50/100/150 pA step, divided by the inverse of the initial interspike interval in that step and subtracted from $100 \%$ (no adaptation $=0 \%)$. The adaptation ratio quantifies the degree of spike adaptation for a given neuron, where neurons with an adaptation ratio of $50 \%$ implies the initial frequency is twice that of the SS frequency. Neurons with an adaptation ratio $>25 \%$ were considered to be adapting. Adaptation analysis was done as previously described (Perry et al., 2015). To visualize spike-frequency adaptation over time, the reciprocal of consecutive interspike intervals is plotted for each AP versus the time after onset of the $1000 \mathrm{~ms}$ current pulse (latency).

For hyperpolarizing current injections $(-150$ to $-50 \mathrm{pA}, 1 \mathrm{~s}, 50 \mathrm{pA}$ increments) instantaneous amplitudes were measured as the maximum negative peak from baseline, whereas SS amplitudes were averaged over the final $100 \mathrm{~ms}$. The absolute sag amplitude corresponds to the difference between instantaneous and SS amplitudes from baseline in response to a $-150 \mathrm{pA}$ step.

APs elicited from depolarizing current pulses (5 pA increments, 10 $\mathrm{ms}$ ) from resting potential, were analyzed for AP and afterhyperpolarization (AHP) parameters: amplitude, half-width (50\% of spike amplitude or $50 \%$ of negative peak amplitude from onset baseline) and rise time (from 10 to $90 \%$ of peak). Traces were baseline corrected after determination of the AP threshold, noted as the point when the increase in membrane potential exceeds $50 \mathrm{mV} / \mathrm{ms}$. Rheobase was defined as the minimum current required to generate a single AP.

For whole-cell voltage-clamp recordings, neurons were clamped at $-60 \mathrm{mV}$ and access resistance compensated by $>66 \%$. Negative voltage steps ( -60 to $-150 \mathrm{mV}, 10 \mathrm{mV}$ increments, $1 \mathrm{~s}$ ) were separated by $3 \mathrm{~s}$ intervals. Current amplitude was measured from baselined traces over the last $200 \mathrm{~ms}$ of the voltage step.

Drug added to the perfusate was as follows: ZD7288 (20 $\mu \mathrm{M}) 10-20$ min before recording.

Fictive locomotion and whole-cell patch-clamp electrophysiology. Fictive locomotion recordings were performed on P0 and P3 mice as described previously (Andersson et al., 2012). Data from left and right L2 roots were grouped for analysis. Fictive locomotion coupled with whole-cell patch-clamp electrophysiology were conducted as described; briefly, spinal cords were dissected in ice-cold cutting solution containing the following (in mM): $130 \mathrm{~K}$-gluconate, $15 \mathrm{KCl}, 0.05 \mathrm{EGTA}, 20 \mathrm{HEPES}, 25$ glucose, with $\mathrm{pH}$ adjusted to 7.4 by $1 \mathrm{M} \mathrm{KOH}$ and equilibrated in aCSF (described Electrophysiology). After dissection the lumbar dorsal horn was removed before equilibration in aCSF. Briefly, the isolated spinal cord was pinned down on its lateral side at thoracic and sacral levels. The dorsal horn of the lumbar spinal cord was carefully removed from segments L2-L 5 by using smooth slicing movements with a fine entomological pin, leaving the ventral horn and ventral roots intact. The cord was then transferred into aCSF held at room temperature $\left(25^{\circ} \mathrm{C}\right)$ and left to equilibrate for $30 \mathrm{~min}$. The spinal cords were placed in the recording chamber and a suction electrode was attached to a free lumbar ventral root. Fictive locomotion was induced by adding a combination of NMDA $(5$ or $7.5 \mu \mathrm{M})+$ serotonin creatinine sulfate monohydrate (5$\mathrm{HT} ; 10 \mu \mathrm{M})+$ dopamine hydrochloride $(50 \mu \mathrm{M})$ to the perfusing aCSF to induce stable locomotor-like output. All chemicals were obtained from Sigma-Aldrich. Recorded ventral root signals containing compound APs were amplified 1000 times, bandpass filtered $(100-10 \mathrm{kHz})$ before being digitized at $20 \mathrm{kHz}$ (Dp-311 Differential Amplifier, Warner Instruments) and recorded using WinEDR (J. Dempster, Strathclyde University) for later off-line analysis. Once cords displayed stable locomotor-like activity for a minimum period of $15 \mathrm{~min}$, Dmrt3-Cre neurons were whole-cell patched (described above) either contralaterally or ipsilaterally to the attached lumbar ventral root. Data from both the ventral root recordings and individual cells were further analyzed. The ventral root data were rectified and low pass filtered using a third-order Butterworth filter with a $5 \mathrm{~Hz}$ cutoff frequency before further analysis. Coherence plots between L2 and L2/L5 traces were analyzed using a Morlet wavelet transform in SpinalCore v1.1 (Mor and Lev-Tov, 2007). Preferential phase alignment across channels (circular plots) and burst parameters were analyzed for at least 20 sequential cycles as previously described (Kjaerulff and Kiehn, 1996) using an in-house designed program in MATLAB (MathWorks R2016a). Additionally, spike-phase histograms were plotted for segments of time with a minimum of 20 sequential ventral root activity bursts by summing the spikes from each whole-cell patched Dmrt3-Cre neuron in 20 degree bins from the extracted phase of the burst-interburst cycle of the ventral root. The von Mises normal circular distribution was calculated in MATLAB using the anglemean and besseli functions, and Spike kernel density estimates were obtained for $1 \mathrm{~s}$ bins by means of the $k$ sdensity function. Kappa values $>0.5$, obtained from the von Mises analysis, were considered significant.

Two-photon microscopy coupled with ventral root electrophysiology. We built a custom designed two-photon laser scanning microscope (2PLSM), in collaboration with the Helmchen Group, University of Zurich, based on the 2PLSM described previously (Göbel and Helmchen, 2007). Briefly, a mode locked Ti:sapphire laser system (Coherent) was used to deliver laser pulses at $100 \mathrm{fs}$ with a repetition rate of $80 \mathrm{MHz}$ at $870 \mathrm{~nm}$ wavelength. The laser intensity was modulated by a Pockel cell (Conoptics) and a telescope was used to focus the beam. Two galvanometric scan mirrors (model 6210, Cambridge Technology) were used to perform 2-D frame scanning ( $x-y$ scanning). A $40 \times$ water-immersion objective [Plan Apochromat; 1 numerical aperture (NA); Zeiss] was used 
and the fluorescence signals emitted were passed through bandpass filters for the red $(610 / 75 \mathrm{~nm})$ and green $(535 / 50 \mathrm{~nm})$ channels, respectively (AHF Analysentechnik) and amplified through two photomultiplier tubes (R6357; Hamamatsu). Signals were sampled at $10 \mathrm{MHz}$ and digitally integrated over pixel dwell times. The microscope was controlled by HelioScan (Langer et al., 2013). Laser power at the focal spot never exceeded $50 \mathrm{~mW}$. Functional images were acquired at $6 \mathrm{~Hz}$ with $128 \times 128$ pixel resolution.

In vitro spinal cord preparation. Dmrt $3^{\mathrm{Cre}} ; t d T$ spinal cords $\mathrm{P} 0-\mathrm{P} 3$ were removed in ice-cold dissection buffer containing the following (in $\mathrm{mM}$ ): $128 \mathrm{NaCl}, 4.69 \mathrm{KCl}, 25 \mathrm{NaHCO}_{3}, 1.18 \mathrm{KH}_{2} \mathrm{PO}_{4}, 3.5 \mathrm{MgSO}_{4}, 0.25 \mathrm{CaCl}_{2}$, and 22 D-glucose; equilibrated with $95 \% \mathrm{O}_{2}$ and $5 \% \mathrm{CO}_{2}$. For hemisection, the spinal cord was pinned down ventral side up and using a fine entomological pin gentle slicing movements were made at the midline, along the length of the spinal cord. Each of the hemisections had the dorsal and ventral roots intact. Dorsal-horn-removed experiments were prepared as described above. Cords were transferred into artificial CSF containing the following (in mM): $128 \mathrm{NaCl}, 4.69 \mathrm{KCl}, 25 \mathrm{NaHCO}_{3}, 1.18$ $\mathrm{KH}_{2} \mathrm{PO}_{4}, 1.25 \mathrm{MgSO}_{4}, 2.5 \mathrm{CaCl}_{2}$, and $22 \mathrm{D}$-glucose; equilibrated with $95 \% \mathrm{O}_{2}$ and $5 \% \mathrm{CO}_{2}$, flow adjusted to $6 \mathrm{ml} / \mathrm{min}$ and maintained at room temperature $\left(25^{\circ} \mathrm{C}\right)$ and left to equilibrate for at least $30 \mathrm{~min}$. Next, neurons were loaded with suitable calcium indicators (see below). Suction electrodes were fitted to flexor-related ventral roots at lumbar segment 2 (L2).

Calcium indicator loading. Two complementary strategies were used to label neurons with calcium indicators for two-photon imaging:

The calcium indicator Oregon Green BAPTA-1 AM (OGB-1 AM; Invitrogen, Life technologies) was dissolved in DMSO/20\% Pluronic F-127 and diluted in Ringer's solution to a concentration of $1 \mathrm{~mm}$ (Johannssen and Helmchen, 2013). Multi-cell bolus loading of calcium indicator dye (Stosiek et al., 2003) was used to label Dmrt3 ${ }^{\mathrm{Cre}}$; $t d T$ neurons in hemisected or dorsal horn removed preparations. A glass micropipette filled with calcium indicator dye was positioned close to the surface of the spinal cord at L2 under video guidance and a $10 \times$ air objective (Plan Apochromat, $0.45 \mathrm{NA}$; Zeiss). In two-photon imaging mode (40× objective), the pipette was then advanced into the tissue at a shallow angle. The dye was pressure-ejected (150-700 mbar for several minutes) at an approximate depth of $60-80 \mu \mathrm{m}$. The spinal cord was incubated with the dye for $1 \mathrm{~h}$.

Combined root stimulation and imaging. Imaging was performed to measure the activity response of Dmrt3 ${ }^{\mathrm{Cre}}$; $t d \mathrm{~T}$ neurons in response to dorsal and ventral root stimulations. The start point and endpoint of the imaging session were indicated by the opening and closing of the shutter from the imaging system and was recorded along with the stimulation artifact. The stimulation artifact was recorded to indicate the onset of stimulation during the imaging period. The stimulus consisted of a pulse train with amplitude of $300 \mu \mathrm{A}$, duration of $2 \mathrm{~s}$, frequency of $20 \mathrm{~Hz}$ and a pulse width of $200 \mu \mathrm{s}$. Calcium imaging was performed on the same set of $\mathrm{Dmrt} 3^{\mathrm{Cre}}$; $t d \mathrm{~T}$ neurons in response to dorsal and ventral root stimulations at the ipsilateral L2. Calcium responses were considered significant if the peak fluorescence was $>2 \times$ the SD from the mean baseline fluorescence. The baseline fluorescence $\left(F_{\mathrm{b}}\right)$ was defined as the fluorescence intensities before the onset of stimulation. The activity response for each neuron was quantified as the percentage change in fluorescence $(\Delta F)$ over the baseline fluorescence $\left(F_{\mathrm{b}}\right): \Delta F / F_{\mathrm{b}}(\%)=\left(\left[F-F_{\mathrm{b}}\right] / F_{\mathrm{b}}\right) \times 100$.

Ventral root electrophysiology. Glass-suction electrodes were connected to the ventral root at L2. Fictive locomotion was induced as described above. Recorded signals were amplified 10,000 times and bandpass filtered from $100 \mathrm{~Hz}$ to $1 \mathrm{kHz}$ (Model EX4-400, Dagan) and digitized at 20 $\mathrm{kHz}$ using a Digidata 1440A AD-converter (Axon CNS, Molecular Devices) and Clampex software (10.2).

Calcium imaging. The L2 was used for all imaging and electrophysiological measurements. After incubation with Oregon green BAPTA, an area is chosen with dual-expressing Dmrt3 neurons (red, Dmrt $3^{\mathrm{Cre}}$; $t d T$ neurons; green, Oregon green BAPTA) for calcium imaging. Each experiment comprised of 9-30 loaded neurons in one field-of-view. The region-of-interest (ROI) for each imaged neuron was manually outlined to extract only the somatic activity profiles. Only neurons with clearly visible somata in the field-of-view were included for analysis. Imaging experiments were performed and data acquired with the software HelioScan (Langer et al., 2013).

A mean projection image from the time lapse imaging data were generated using the image processing program ImageJ. The mean projection image has sharper quality and aids in better identification of cellular somata for demarcation of the ROI. After background subtraction, the mean fluorescence intensities per ROI /frame were extracted for the entire time-lapse imaging series. The fluorescence time-series was extracted for all ROIs in the field-of-view. Imaging data were analyzed using IGOR Pro (WaveMetrics), MATLAB (MathWorks) and MS Excel (Microsoft). Fluorescence time-series data extracted from the neurons in the field-ofview was smoothened using the box smoothing function in IGOR Pro. We defined the baseline $\left(F_{\mathrm{b}}\right)$ as the 10th percentile value of the fluorescent intensities for the respective neuron. The activity response for each neuron was quantified as the percentage change in fluorescence $(\Delta F)$ over the baseline fluorescence $\left(F_{\mathrm{b}}\right): \Delta F / F_{\mathrm{b}}(\%)=\left(\left[F-F_{\mathrm{b}}\right] / F_{\mathrm{b}}\right) \times 100$.

Frequency analysis in calcium imaging data. Time periods corresponding to peaks of interneuron bursting with amplitudes over baseline activity was determined $(\Delta F / F)$. The frequencies of oscillation in individual Dmrt3-Cre interneurons were calculated as the number of bursts produced per time unit (frequency $=$ no. of bursts/duration of imaging). For analysis of burst variability, the interburst intervals were determined for every successive burst pairs. The instantaneous frequency was defined as the inverse of the interburst interval.

Experimental design and statistical analysis. All statistical analyses were done using GraphPad Prism Software or MATLAB (MathWorks). Electrophysiological recordings, including passive and active properties and $I_{\mathrm{h}}$, and normalized interburst intervals and correlation coefficient from two-photon recordings were analyzed using student's unpaired twotailed $t$ test assuming unequal variance, a one-way ANOVA, or the twotailed Mann-Whitney test. Ventral root recording preferential phase alignment was assessed by means of circular statistics (Rayleigh $t$ test) for 20 consecutive cycles as described by Kjaerulff and Kiehn (1997). Burst parameters and locomotor frequency were compared using the twotailed Mann-Whitney test. Twelve mouse pups were used in slice patchclamp experiments; three animals were used in whole spinal cord preparations without fictive locomotor drugs and seven pups were used in whole spinal cord populations with fictive locomotor drugs. Mice of either sex were used in this study. The significance was determined as ${ }^{*} p<0.05,{ }^{* *} p<0.01,{ }^{* *} p<0.001$.

\section{Results}

\section{Generation of a Dmrt $3{ }^{\mathrm{Cre}}$ mouse line to target Dmrt3 interneurons}

To characterize Dmrt3-derived spinal interneurons, we generated a Cre knock-in mouse line using the ZFN technology (Sigma-Aldrich; Meyer et al., 2010). ZFN mRNAs designed for the Dmrt3 locus, and a donor vector carrying iCre and $2 \mathrm{~A}$ peptide sequence were introduced to B6D2F1 zygotes by pronuclear microinjection. ZFNs directed toward Dmrt3 exon 1 allowed for genomic DNA cleavage followed by site-specific recombination of iCre at the ATG site of Dmrt3. Founder mice were screened for correct Cre insertion and the expression pattern was investigated

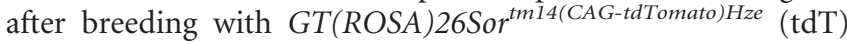
reporter mice to create Dmrt3 ${ }^{\mathrm{Cre}} ; \mathrm{R}_{2} 6^{\text {tdTomato }}\left(\mathrm{Dmrt} 3^{\mathrm{Cre}} ; \mathrm{tdT}\right)$ mice (Fig. 1 and Fig. 1-1, available at https://doi.org/10.1523/ JNEUROSCI.0326-18.2018.f1-1). IHC confirmed that $84 \%$ of the Dmrt3 ${ }^{C r e} ; t d T$ marked cells expressed DMRT3 $(n=507)$ at embryonic day (E) 12.5. However, 2 d later (E14.5), we found that $74 \%(n=342)$ of the Dmrt3 ${ }^{\text {Cre }} ; t d T$ marked cells expressed DMRT3 (Fig. 1A). Vice versa, 99.6\% (252/253, 10 ventral horns from 3 embryos, 3-4 ventral horns/embryo) of the DMRT3 immunolabeled cells colocalized with Dmrt3 ${ }^{\mathrm{Cre}} ; \mathrm{tdT}$ neurons. $D m r t 3$, similar to other transcription factors, has a restricted and well characterized temporal expression pattern during spinal cord development. Dmrt3-positive neurons increase in number 
A
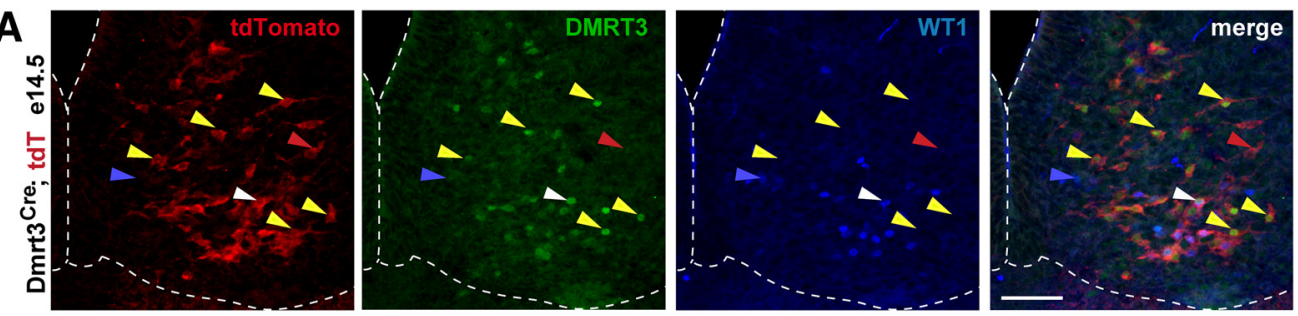

B
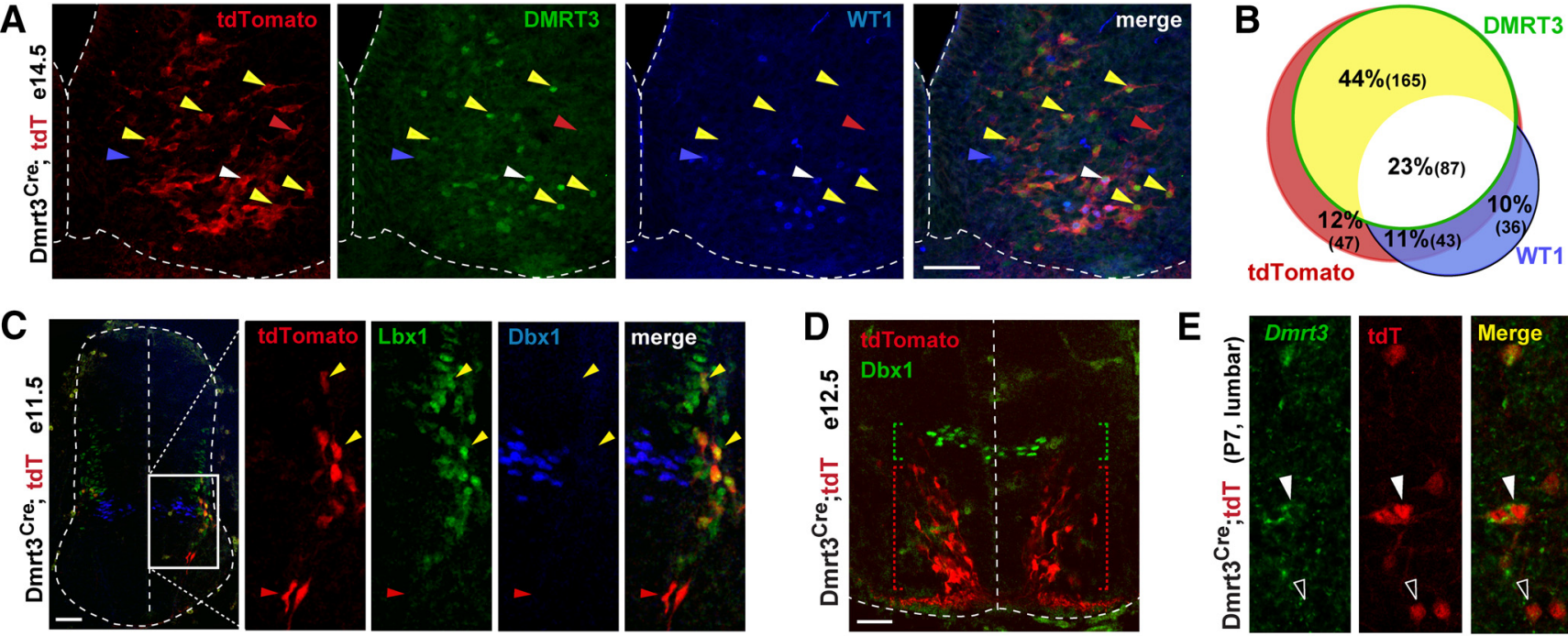

$\mathrm{E}$
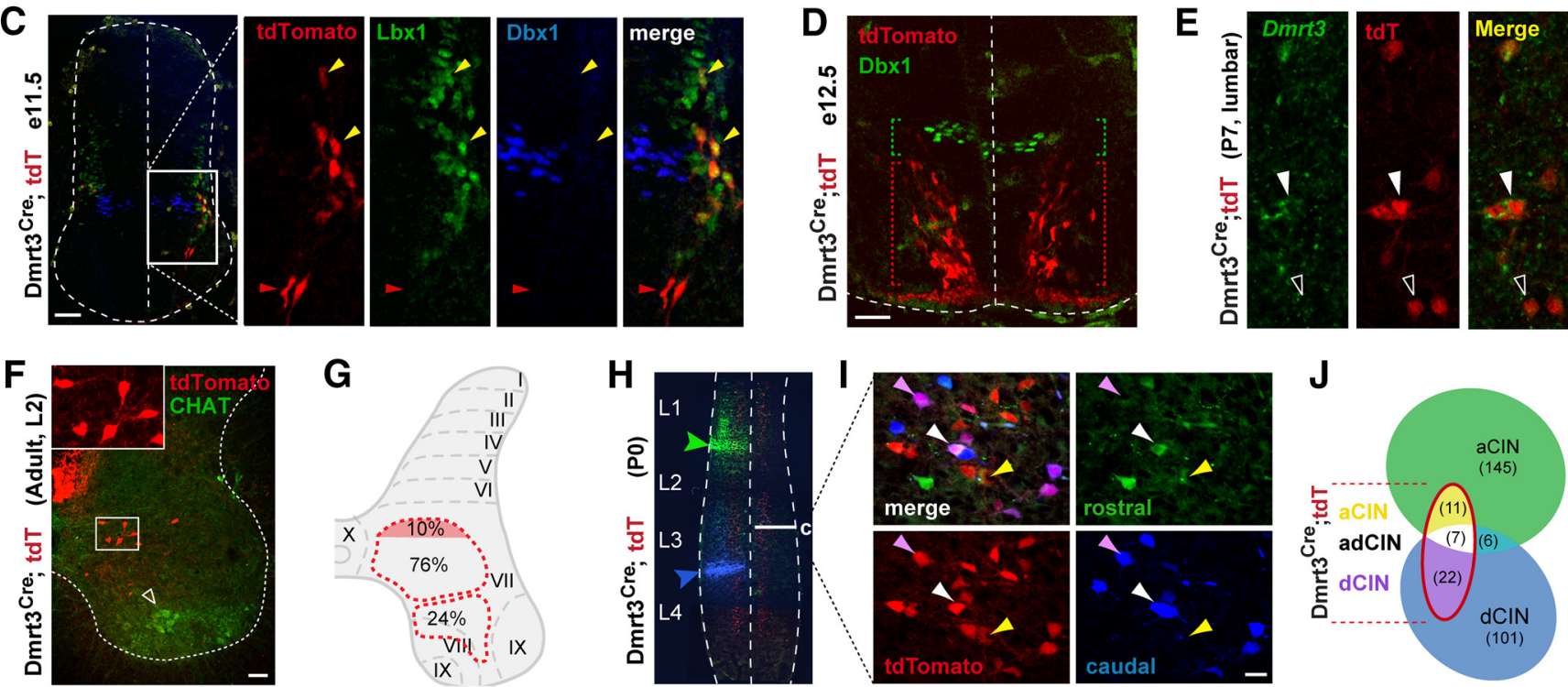

Figure 1. Anatomical and molecular characterization of the Dmrt3-derived interneuron population, genetically targeted by the Dmrt3 ${ }^{\text {Cre }}$ mouse line. $A$, DMRT3 ${ }^{+}$immunolabeled neurons (green) colocalized with Dmrt3 $3^{\text {Cre }}$; $t d T$ neurons (red) in E14.5 mouse spinal cords (yellow arrows). The majority of the Dmrt3 ${ }^{\text {Cre }} ;$ tdT neurons expressed DMRT3 protein (B, yellow and white regions). Some Dmrt3 ${ }^{\text {Cre }}$,tdT/ DMRT3 ${ }^{+}$marked neurons also coexpressed another dl6-neuron marker WT1 (blue, white arrows). Single-labeled tdT ${ }^{+}$and WT1 ${ }^{+}$neurons are indicated by red and blue arrows, respectively. Scale bar, $50 \mu \mathrm{m} . n=10$ ventral horns $/ 3$ embryos. $\boldsymbol{B}$, Venn diagram displaying the proportion of Dmrt3 ${ }^{\text {(re }}$; $t d T$ neurons colocalizing with DMRT3 and WT1 marked cells. The percentage of each neuron group is given in relation to all labeled neurons (Dmrt3 ${ }^{\text {(re }}$; $t d T$ and WT1 ${ }^{+}$and DMRT3 ${ }^{+}$, in total 378). C, Lbx ${ }^{+}$-immunolabeled neurons (green) colocalized with most of the Dmrt3 $3^{\text {Cre }}$; $t d T$ neurons (red) in E11.5 mouse spinal cords. A subset of Dmrt ${ }^{\text {(re }}$; $t d T$ neurons expressing Lbx1 are localized dorsally to the Dbx1 immunolabeled neurons (blue) $S c a l e$ bar, $50 \mu \mathrm{m}$. $D$, Dbx1 ${ }^{+}$-immunolabeled neurons (green) and Dmrt3 ${ }^{\text {(re }}$; tdT neurons (red) in E12.5 mouse spinal cords. Scale bar, $50 \mu \mathrm{m}$. E, ISH for Dmrt3 mRNA (green) colocalized with Dmrt3 ${ }^{\text {(re }}$; tdT (red) marked cells at P7 in lumbar spinal cord cross sections (yellow, closed arrow). Some tdT-positive cells were negative for Dmrt3 mRNA (open arrow). $\boldsymbol{F}$, Photomicrograph of L2 (16 $\mu \mathrm{m})$ from adult Dmrt ${ }^{\text {(ree }}$; $t d T$ spinal cord showing the position of Dmrt3-Cre neurons (red) in relation to motor neurons (ChAT immunohistochemistry; green, open arrow). Inset, Higher-magnification of the Dmrt3 ${ }^{\text {(re }}$; $t d T$ cells (red). Scale bar, $100 \mu \mathrm{m} . n=12$ ventral horns/3 mice. G, Dmrt3 ${ }^{\text {(re }}$; $t$ tdT cells were located in the intermediate zone of the spinal cord in Rexed Laminae VII/VIII and positioned medially (76\%), ventrally (24\%), and a small portion (10\% of the dorsal portion) dorsally of the central canal. Data collected from the lumbar spinal cord. $\boldsymbol{H}$, Retrograde FDA tracers applied at lumbar level L 1 (green) and between L3 and L4 (blue) in Dmrt3 ${ }^{\text {(re }}$; $t d T$ spinal cords. $\boldsymbol{I}$, Transverse spinal cord section on the contralateral side (c; indicated by white line in $\boldsymbol{H}$ ) illustrating $D \mathrm{mrt} 3^{\text {(re }}$; $t d T$ neurons (red; $n=40$ ) with ascending commissural interneurons (aCIN; 11/40; yellow arrow), descending (dCIN; 22/40; magenta arrow,) and bifurcating Dmrt3 ${ }^{\text {(re }}$; tdT fibers (adCIN; 7/40; white arrow,). Scale bar, $10 \mu \mathrm{m}$. Data were obtained from $n=6$ neonatal mice. J, Quantification of traced commissural neurons and the proportion of Dmrt3 $3^{\text {(re }} ; t d T$ neurons (red oval region). See also Figure 1-1, available at https://doi.org/10.1523/JNEUROSCI.0326-18.2018.f1-1.

between E12.5 and E14.5, and later decline before postnatal day (P)4, providing a likely explanation for these additional Dmrt3 ${ }^{\text {Cre }} ;$ tdT-positive neurons (Andersson et al., 2012).

To examine the origin of Dmrt $3^{\mathrm{Cre}} ; t d T$ cells, we performed a staining on E11.5 spinal cord with both an anti-Lbx1 antibody that labels the dI4 to dI6 interneuron populations and an antiDbxl antibody that labels V0 progenitors. At E11.5 most of the $D m r t 3^{C r e} ; t d T$ neurons just started their migration and coexpressed Lbx1. Moreover, some of these cells were localized above the Dbx1 domain suggesting that they do not arise from the V0 progenitors (Fig. 1C). At E12.5, most of the Dmrt3 ${ }^{\mathrm{Cre}}$; $t$ t T neurons have already migrated ventrally (Fig. 1D). Further, we found a decrease in Dmrt3 mRNA expression and in situ mRNA signal after birth, suggesting that Dmrt3 expression is downregulated after differentiation (Fig. $1 E$ and Fig. 1-1, available at https://doi. org/10.1523/JNEUROSCI.0326-18.2018.f1-1).

In addition to the Dmrt3 population in the spinal cord, Dmrt $3^{\mathrm{Cre}} ; t d \mathrm{~T}$ expression was found in the brain, predominantly in neurons in the cerebral cortex with dense arborization (Fig. 1-1, available at https://doi.org/10.1523/JNEUROSCI.0326-18. 2018.f1-1). These observations are in line with previous studies reporting Dmrt3 expression in the telencephalon, neural tube and additional tissue including the gonads and spinal cord in both chicken and mouse (Smith et al., 2002; Kim et al., 2003; Ahituv et al., 2007; Zagoraiou et al., 2009). Spinal cord Dmrt3 neurons expressed VIAAT and are thus inhibitory, however, the character of cortical Dmrt3 neurons remains unknown. Singlecell RT-PCR on randomly selected $\mathrm{Dmrt} 3^{\mathrm{Cre}} ; \mathrm{tdT}$ neurons from the primary motor cortex of adult mice revealed that $33 \%(11 / 33)$ were inhibitory based on the expression of GAD67 (Fig. 1-1, available at https://doi.org/10.1523/JNEUROSCI.0326-18.2018. f1-1), whereas the remaining $66 \%(22 / 33)$ were presumably excitatory neurons (Fig. 1-1, available at https://doi.org/10.1523/ JNEUROSCI.0326-18.2018.f1-1). Thus, the Dmrt3 neurons in the motor cortex comprise a mixed population of inhibitory and excitatory cells. 
WT1, another dI6 interneuron marker shown to partially overlap with DMRT3, marked 35\% (87/252) of DMRT3 ${ }^{+}$/ Dmrt3 ${ }^{\mathrm{Cre}} ; t d \mathrm{~T}$ colabeled cells and $38 \%(130 / 342)$ of the whole $D m r t 3^{\mathrm{Cr}} ; t d \mathrm{~T}$ population. Further, we observed some Dmrt3 ${ }^{\mathrm{Cre}}$; $t d T$-expressing cells that did not colocalize with DMRT3 protein $(26 \%, 90 / 342)$ at E14.5, some of which expressed WT1 (48\%, 43/90; Fig. $1 A, B)$. In addition to marking DMRT3-expressing spinal cord interneurons, the $\mathrm{Dmrt}^{\mathrm{Cre}}$; $t d \mathrm{~T}$ mouse line revealed rostro-caudal projections located in the ventral part of the dorsal funiculus (Fig. $1 F$ and Fig. 1-1, available at https://doi.org/ 10.1523/JNEUROSCI.0326-18.2018.f1-1). The Dmrt3 ${ }^{\text {Cre }} ; t d T$ marked corticospinal tract was not present in embryonic spinal cords and only appeared at $\sim \mathrm{P} 7$, which is the time point when the corticospinal tract reaches the lumbar enlargement in mice (Joosten et al., 1995). Together, the Dmrt3 ${ }^{\text {Cre }}$ knock-in mouse line targets in principle all Dmrt3-derived neurons. A smaller subset of the Dmrt $3^{\mathrm{Cre}}$; $t d \mathrm{~T}$ labeled neurons are unaccounted for, and although it is quite likely that they derive from neurons that have previously expressed Dmrt3, we herein refer to the population as Dmrt3-Cre neurons for clarity.

\section{Dmrt3-Cre neurons are positioned in laminae VII and VIII and send both ascending and descending commissural projections}

During embryogenesis, dI6 neurons are born dorsally and migrate ventrally, finally settling in Rexed laminae VII and VIII (Andersson et al., 2012; Vallstedt and Kullander, 2013). We characterized the distribution of adult Dmrt3-Cre neurons at cervical, thoracic and lumbar levels (Fig. 1 and Fig. 1-1, available at https:// doi.org/10.1523/JNEUROSCI.0326-18.2018.f1-1). The majority of $\mathrm{Dmrt3}{ }^{\mathrm{Cre}}$; $t d \mathrm{~T}$-expressing neurons were located in the intermediate zone in laminae VII $(76 \%, n=149 / 197$; Fig. $1 F, G ; 16$ ventral horns from 3 adult mice), whereas some Dmrt3-Cre cells (24\%, $n=48 / 197)$ were positioned farther into the ventral horn and distributed to laminae VII and VIII $(>300 \mu \mathrm{m}$ from the central canal, $<300 \mu \mathrm{m}$ from the ventral rim). A small fraction of the medially located cells in laminae VII $(10 \%, n=19 / 197)$ were located dorsal of the central canal (Fig. $1 G$ ). The majority of the Dmrt3-Cre population is comprised of neurons with a soma diameter of $10-15 \mu \mathrm{m}$, however, some larger neurons $(>20 \mu \mathrm{m}$ diameter) were observed $(13 \%, 47 / 349)$. The ratio of $\mathrm{Dmrt3}^{\mathrm{Cre}}$; $t d T$ neurons to immunolabeled choline acetyl transferase (ChAT) motor neurons was $\sim 1: 1$ (104:95, $n=12$ ventral horns, 3 mice; Fig. $1 F$ and Fig. 1-1, available at https://doi.org/10.1523/ JNEUROSCI.0326-18.2018.f1-1).

In neonates, dI6 neurons, including the Dmrt3 population, are inhibitory and can project both contralaterally and ipsilaterally (Lanuza et al., 2004; Rabe et al., 2009; Andersson et al., 2012). Here, we observed adult $\mathrm{Dmrt}^{\mathrm{Cre}}$; $t d \mathrm{~T}$ fibers projecting over the midline in the ventral funiculus (Fig. 1-1, available at https:// doi.org/10.1523/JNEUROSCI.0326-18.2018.f1-1). Commissural interneurons can be classified according to their axonal projections as ascending, descending or bifurcating (Eide et al., 1999). To further examine Dmrt3-Cre axonal projection patterns, retrograde dextran tracers were applied at lumbar level L1 and between L3and L4 roots of Dmrt3 ${ }^{\mathrm{Cre}} ; t d T$ spinal cords (P0 and P1, 6 spinal cords; Fig. $1 H$ ). We found that $28 \%$ of the commissural L2-L3 Dmrt ${ }^{\mathrm{Cre}}$; $t d \mathrm{~T}$ neurons were ascending (11/40), 55\% descending (22/40), and 18\% (7/40) were bifurcating (Fig. $1 I, J$ ).

\section{Dmrt3-Cre neurons receive diverse synaptic inputs and provide inhibitory contacts onto motor neurons}

We next investigated the synaptic inputs onto Dmrt3-Cre neurons (Fig. 2A). Immunohistochemistry for vesicular neurotransmitter transporters [Vesicular Glutamate Transporter (VGLUT)1 and 2; SLC17A7 and SLC17A6] combined with synaptophysin (SYP), suggests that $\mathrm{Dmrt}{ }^{\mathrm{Cr}}$; $t d T$ neurons receive excitatory inputs. In particular, at $\mathrm{P} 4$, we found $\mathrm{Dmrt} 3^{\mathrm{Cre}}$; $t d \mathrm{~T}$ cells in the midst of VGLUT1-immunopositive terminals, and synaptophysin/ VGLUT1-positive puncta on Dmrt3 ${ }^{\text {Cre }}$; $t d T$ cells (Fig. $2 B$ ). At 4-6 weeks of age, we found abundant VGLUT1 and VGLUT2immunopositive terminals double labeled with synaptophysin on Dmrt3 ${ }^{\text {Cre }}$; $t d T$ cells (Fig. 2C,D). Glutamatergic sensory neurons may innervate locomotor related interneurons (Alvarez et al., 2004), and for that reason, the possible activation of Dmrt3 ${ }^{\mathrm{Cre}} ; t d \mathrm{~T}$ cells by sensory glutamatergic terminals was investigated by dorsal root stimulations. Dmrt3-Cre neurons responded to dorsal, but not ventral, root stimulations ( $n=19$; Fig. $2 E$ ). This asymmetry suggests that Dmrt3 neurons are regulated by sensory afferent neurons, but not by motor collateral axons. Further, we observed synaptophysin-immunopositive puncta also immunopositive for VIAAT, (SLC32A) suggestive of inhibitory inputs, as well as occasional monoaminergic synapses [SLC18A2, vesicular monoamine transporter (VMAT2), presumably deriving from monoaminergic nuclei in the brainstem; Fig. $2 F, G]$. We also found immunopositive cholinergic synapses [SLC18A3, vesicular acetylcholine transporter (VAChT)], likely originating from local interneurons, such as partition cells or V0c cells, on Dmrt $3^{\mathrm{Cre}}$; $t d \mathrm{~T}$ neurons (Fig. $2 \mathrm{H}$ ). In further support, we found synapses marked by SLC10A4 (solute carrier family 10 member 4), an orphan vesicular transporter highly expressed in aminergic neurons (Larhammar et al., 2015), suggesting that Dmrt3-Cre cells receive cholinergic and/or monoaminergic input (Fig. $\left.2 I, I^{\prime}, I^{\prime \prime}\right)$.

Dmrt3-positive neurons, as previously identified by immunolabeling, were found to be inhibitory and innervate motor neurons (Andersson et al., 2012). Using Dmrt3 ${ }^{\mathrm{Cre}}$; $t d T$ mice and RNA in situ hybridization (Fig. 1-1, available at https://doi.org/10. 1523/JNEUROSCI.0326-18.2018.f1-1), we found that $85 \%$ of the Dmrt3-Cre neurons colocalized with Viaat and 0\% with Vglut2 mRNA. Further, glycine immunolabeling colocalized with the majority of Dmrt3 ${ }^{\mathrm{Cre}}$; $t d T$ marked neurons in adult mice (65\%; Fig. 1-1, available at https://doi.org/10.1523/JNEUROSCI.0326-18.2018. f1-1). We also found vesicular inhibitory amino acid transporter (VIAAT, SLC32A1) -positive terminals to colocalize with tdT on ChAT immunolabeled motor neurons (Fig. 2J), corroborating the presence of inhibitory contacts on motor neurons. This demonstrates that the majority (if not all) spinal Dmrt3-Cre neurons are inhibitory and primarily glycinergic.

Dmrt ${ }^{\mathrm{Cre}} ; t d T$ terminals were observed on cholinergic neurons close to the central canal (Fig. $2 \mathrm{~K}$ ), presumably V0c interneurons (Zagoraiou et al., 2009). Dmrt ${ }^{\text {Cre }}$; $t d T$ terminals were also found on Calbindin-immunolabeled neurons near the ventral rim, which suggest that dI6 neurons innervate Renshaw cells (Fig. 2L). In summary, the Dmrt3-Cre population extends inhibitory commissural fibers across several spinal cord segments along the rostrocaudal axis, synapse onto motor neurons, Renshaw cells, V0c cells, and receives excitatory, inhibitory, and modulatory inputs (Fig. 2A). Thus, Dmrt3 neurons receive inputs from supraspinal neurons presumably in the brainstem, and from sensory, but not motor components, and are therefore suitably positioned to integrate and relay information to premotor and motor neuron circuits. 
A

E
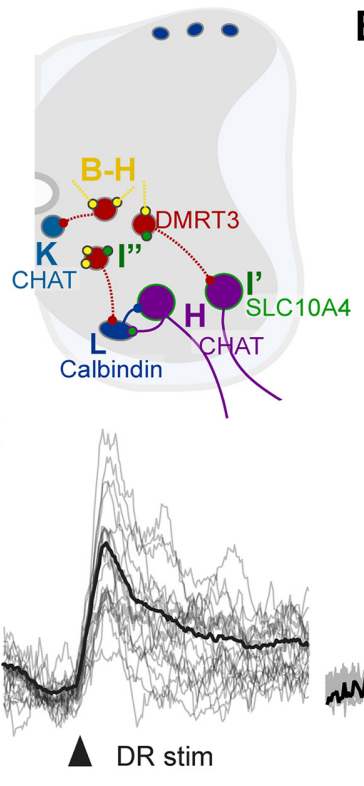

B
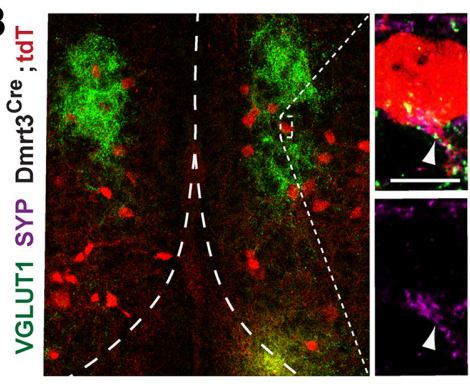

F

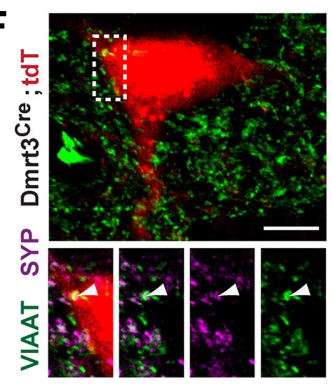

C

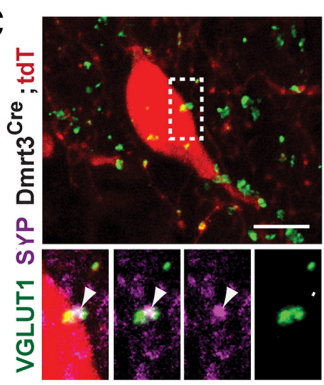

G

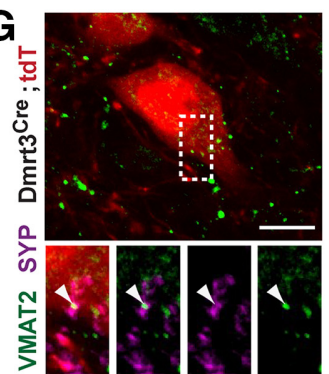

D
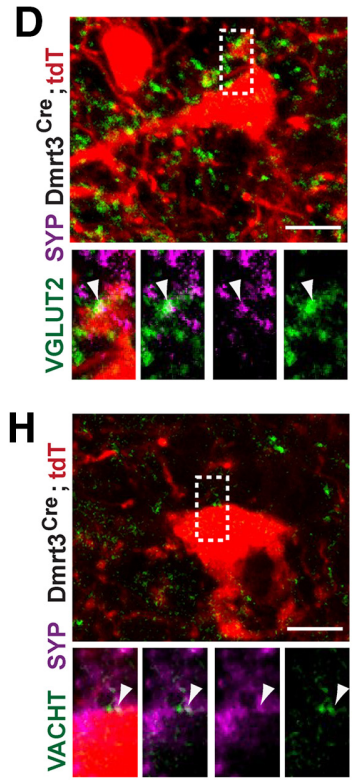
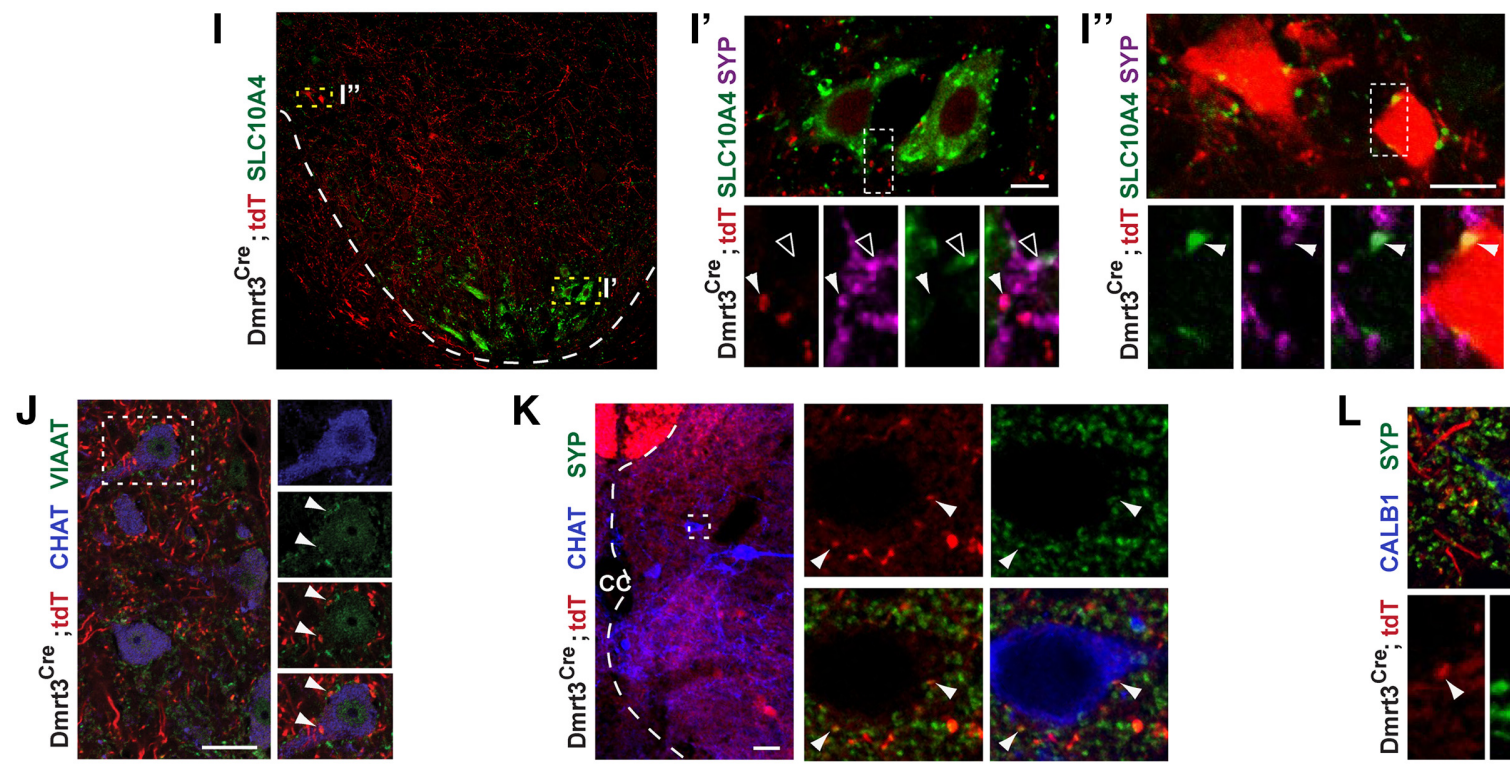

K

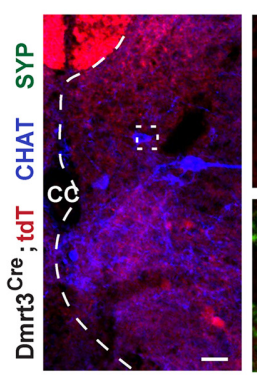

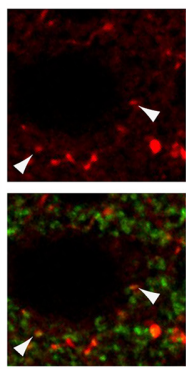
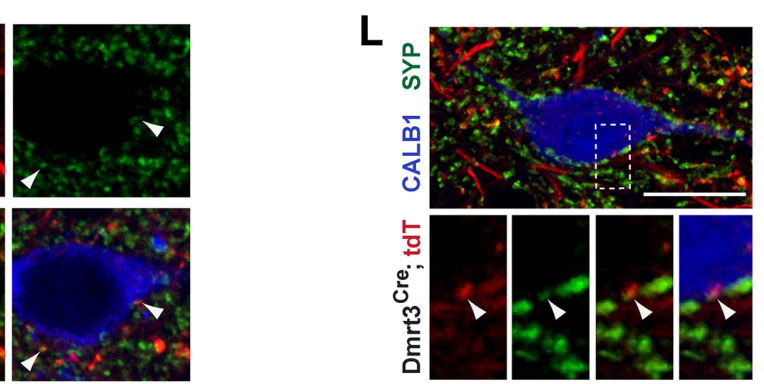

Figure 2. Connectivity characterization of the Dmrt3-Cre-derived interneurons. $A$, Schematic overview of connections to and from Dmrt3-Cre cells presented in the figure. $\boldsymbol{B}$ - $\boldsymbol{L}$, Dmrt $3^{\text {Cre }}$; $t d T$ neurons (red) receive excitatory and inhibitory synaptic terminals, as well as cholinergic and monoaminergic contacts (green). Synaptic terminals identified with SYP (magenta). Arrows point at individual synaptic terminals (white) present on Dmrt3 ${ }^{\text {rre }}$, $t d T$ neurons (red). Boxed areas show higher-magnification panels of separated channels. B-D, Glutamatergic terminals were immunolabeled for VGLUT1 at P4 (B) and VGLUT1 and VGLUT2 at 4 weeks ( $\boldsymbol{C}, \boldsymbol{D}$; avg 5.8 puncta/cell, also immunopositive for SYP). $\boldsymbol{E}$, Calcium activity response of a Dmrt3-Cre neurons ( $n=19)$ to stimulations through the dorsal (DR) and ventral (VR) ipsilateral L2 root (gray trace, individual response; black trace, average response). $\boldsymbol{F}$, Inhibitory synapses were immunolabeled for VIAAT (avg 6.9 puncta/cell). G, Monoaminergic terminals immunolabeled for VMAT2 (0.34 puncta/cell). $\boldsymbol{H}$, Cholinergic terminals immunolabeled for VACHT (0.5 puncta/cell). Scale bars: $\boldsymbol{B}-\boldsymbol{H}, 25 \mu \mathrm{m} . n=6-8$ Dmrt3 $3^{\text {Cre }}$; $t d T$ neurons. $I-I^{\prime \prime}, D m r t 3^{\text {Cre }}, t d T$ neuron innervation onto motor neurons was confirmed by colocalization of Dmrt ${ }^{\text {Cre }}$; $t d T$ (red) SYP puncta (magenta) to the cholinergic motor neuron marker SLC10A4 (green). SLC10A4 immunolabeled synapses (green/magenta) were also observed on Dmrt3 ${ }^{\text {(ree }}$; tdT marked neurons (red). Scale bars: I, $100 \mu \mathrm{m}, I^{\prime}, I^{\prime \prime}, 10 \mu \mathrm{m} . J$, Motor neurons identified by ChAT immunohistochemistry (blue) displayed inhibitory tdTomato boutons likely arising from Dmrt3 ${ }^{\text {(ree }}$, tdT-derived spinal interneurons (red), illustrated by colocalization (arrows, yellow) with the VIAAT (SLC32A1, green). Scale bar, $25 \mu \mathrm{m}$. $\boldsymbol{K}$, Cholinergic neurons located close to the central canal, labeled by ChAT IHC (blue), displayed inhibitory tdTomato boutons likely arising from $D_{m r t 3}{ }^{\text {Cre }}$; $t d T$-derived spinal interneurons (red), illustrated by colocalization (arrows, yellow) with SYP (green). Avgerage 2.0 puncta/cell also positive for SYP, $n=45$ Dmrt3 ${ }^{\text {Cre }} ; t d T$ neurons. Scale bar, $25 \mu \mathrm{m}$. L, Ventral rim calbindin-immunopositive neurons (blue) were positive for inhibitory tdTomato boutons likely arising from Dmrt3 ${ }^{\text {(re }}$, tdT-derived spinal interneurons (red), illustrated by colocalization (arrows, yellow) with SYP (green). Avgerage 2.1 puncta/cell also positive for SYP. Scale bar, $25 \mu \mathrm{m} . n=34$ Dmrt $3^{\text {cre }}$; $t d T$ neurons. See also Figure 1-1, available at https://doi.org/10.1523/JNEUROSCI.0326-18.2018.f1-1.

Electrophysiological properties of Dmrt3-Cre neurons

We examined the electrical properties of the Dmrt ${ }^{C r e}$; $t$ TT neurons, extending the characterization of the dI6 population to medial and ventral regions of the lumbar spinal cord, where patched neurons were filled with biocytin to show their morphology and confirm their position within the spinal cord (Fig. 3-1, available at https://doi.org/10.1523/JNEUROSCI.0326-18.2018. f3-1). Dmrt3-Cre neurons in the intermediate area of lamina VII and VIII close to the central canal had an average resting membrane potential of $-53.8 \pm 0.87 \mathrm{mV}$, input resistance of $431.6 \pm$ $35.5 \mathrm{M} \Omega$, and whole-cell capacitance of $13.7+/-1.2 \mathrm{pF}(n=$ 42 ), and fired APs in response to depolarizing pulses ( $5 \mathrm{pA}$ incre- 
A
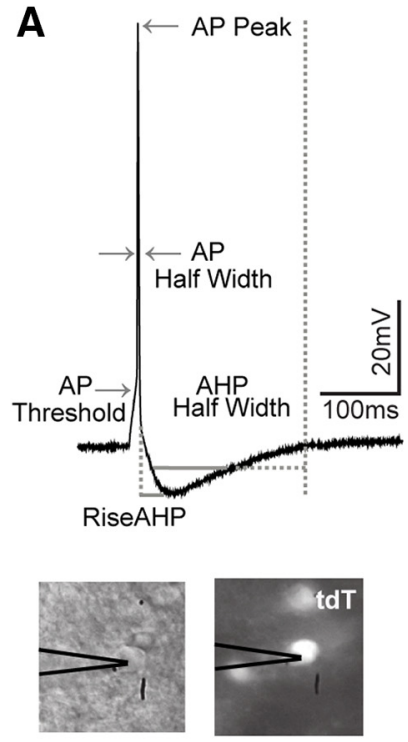

B Dmrt3 ${ }^{\mathrm{Cre}}$ țdT

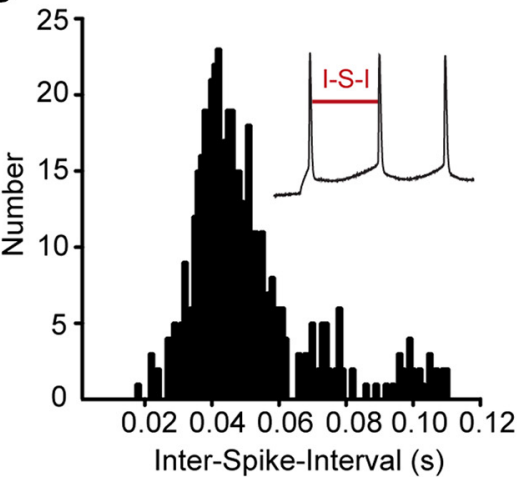

f/I Relationship
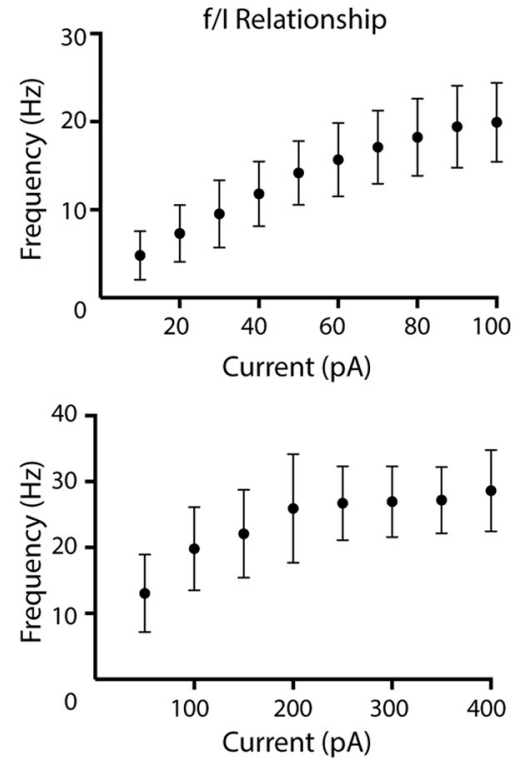

Spike-frequency Adaptation

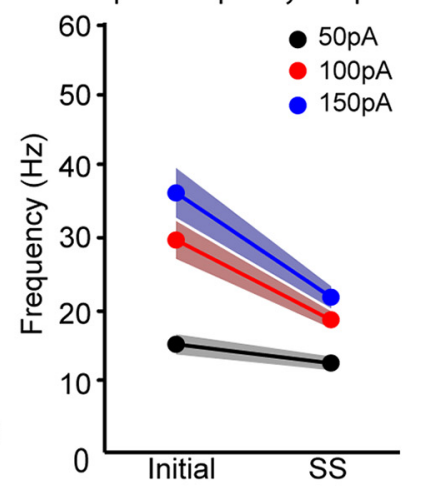

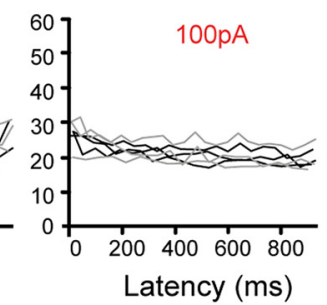
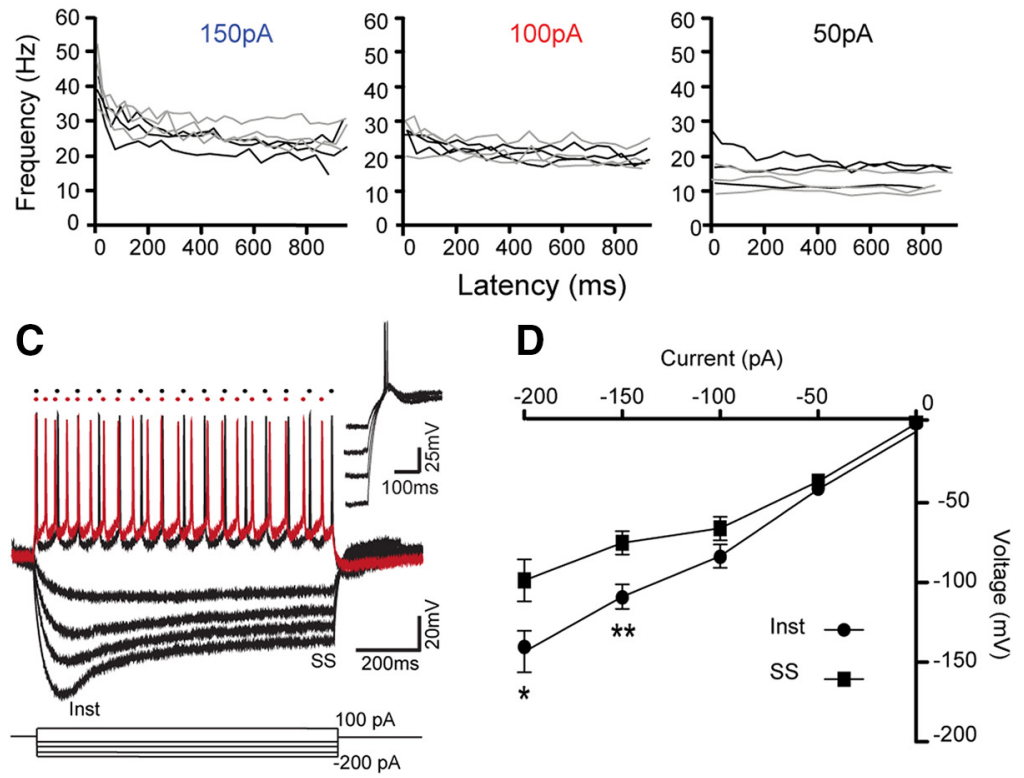

Figure 3. Electrophysiological properties of Dmrt3 interneurons. $\boldsymbol{A}$, Left, Representative Dmrt3 ${ }^{\text {Cre }}$; $t d T$ AP showing prominent AHP. Analysis parameters depicted by solid and dashed gray lines. Whole-cell patch-clamped Dmrt ${ }^{3(r e}, t d T$ cells visualized under bright field (left) and fluorescent (right) light. Black lines denote recording pipette. Right, Frequency (f) versus current $(I)$ plots show the firing frequency of Dmrt3-Cre neurons in response to $10 \mathrm{pA}$ (top plot) and $50 \mathrm{pA}$ (bottom plot) depolarizing steps. Data represented as mean \pm SD. $\boldsymbol{B}$, Left, Interspike interval (I-S-I) histogram for all recorded Dmrt $3^{\text {(re }}$; $t d T$ cells showing the distribution for the population in response to a $100 \mathrm{pA}$ step (bin size $=1 \mathrm{~ms} ; n=31$ ). Right, The relationship between the initial and SS firing frequency for each neuron to $50 \mathrm{pA}$ (black circles), $100 \mathrm{pA}$ (red circles), and $150 \mathrm{pA}$ (blue circles) steps demonstrate the adaptation

ments, 10 ms duration; Fig. $3 A$; Table 1$)$. Neurons from newborn (P0-P2, $n=16)$ and 1-week-old Dmrt3-Cre mice (P6-P7, $n=20)$ had similar active and passive membrane properties (Table 1; spike rate P0-P2 vs P6-P7; Mann-Whitney $U=28$, $n_{(1)}=14, n_{(2)}=15, p=0.0004$, twotailed), suggesting that these cells are mature at birth and likely only undergo minor changes during the first postnatal week. Similar to previous recordings in commissural inhibitory and V0/dI6 neurons (Wu et al., 2011; Dyck et al., 2012), a proportion of Dmrt3 ${ }^{\mathrm{Cre}}$; $t$ dT neurons (20/ 42) exhibited spontaneous firing at rest, in the absence of injected current. Dmrt3-Cre neurons were not further discriminated based on anatomical position, because the electrical properties of Dmrt3-Cre neurons recorded dorsally $(0-100 \mu \mathrm{m}, 49 \%, 16 / 33)$, medially $(33 \%, 11 / 33)$, or ventrally $(18 \%$, $6 / 33$ ) in the intermediate zone in laminae VII were similar (Table 2).

Dmrt3-Cre neurons increased their firing frequency in response to increasing depolarising current steps (Fig. $3 A ; 10$ or $50 \mathrm{pA}$ increments, $1 \mathrm{~s}$ duration) and exhibited spike-frequency adaptation where neurons exhibited a slower, more regular firing frequency toward the end of the current step (Fig. 3B). This adaptation was more prominent at higher current inputs where $68 \%(100 \mathrm{pA})$ and $90 \%(150 \mathrm{pA})$ of the Dmrt3-Cre neurons adapted, with a mean adaptation (adaptation index (Haddad and Getting, 1989; Kinnischtzke et al., 2012) of 37 and $40 \%$, respectively. At lower current inputs (50 pA), 46\% (14/31) of neurons exhibited adaptive firing where the mean adaptation was $17 \%$. Thus, most Dmrt3-Cre neurons had substantial spike adaptation at higher frequencies. An agedependent difference between Dmrt3-Cre neurons (P0-P2, $n=16$; P6-P7, $n=20$ ) was not observed (Fig. 3B).

\section{$\leftarrow$}

by neurons at higher current inputs. Circles depict average firing frequency; shaded area depicts the SEM. Graphs below show representative age examples (3 per age in each plot) of ${ }_{\text {Dmrt3 }}{ }^{\text {(re }}$, td T spike-frequency adaptation over the $1000 \mathrm{~ms}$ step (P0-P2, black, $n=16:$ P6-P7, gray, $n=20)$. C, Example voltage trace to depolarizing $(100 \mathrm{pA})$ and hyperpolarizing ( 0 to $-200 \mathrm{pA}$ ) current steps, shows regular AP firing and large depolarizing membrane sag (black, $n=20$ ). Example trace of an accommodating neuron (red) in response to the same depolarization (100 pA) is superimposed. Dots above traces indicate spikes. Rebound APs were occasionally present (inset; $n=11$ ). $\boldsymbol{D}$, A graph illustrating the current-voltage relationship between instantaneous (Inst) and SS membrane potentials in response to hyperpolarization steps $(0$ to -200 $\mathrm{pA})$. Data represented as mean \pm SEM unless otherwise stated. ${ }^{*} p<0.05,{ }^{* *} p<0.01$. See also Figure 3-1, available at https://doi.org/10.1523/JNEUROSCI.0326-18.2018.f3-1. 
Table 1. Membrane and firing properties of $\mathrm{Dmrt} 3^{\mathrm{Cre}}$; $t \mathrm{t} T$ interneurons

\begin{tabular}{|c|c|c|c|c|c|}
\hline & Overall, P0 -P7 & $P 0-P 2, d$ & P6-P7, d & $\mathrm{Dmrt3}^{\mathrm{Cre}} I_{h}$ & Dmrt3 ${ }^{\mathrm{Cre}}$ \\
\hline$n$ & 42 & 16 & 20 & 18 & 11 \\
\hline Input resistance, $\mathrm{M} \Omega$ & $431.57 \pm 35.46$ & $499.38 \pm 64.74$ & $412.60 \pm 49.59$ & $404.89 \pm 46.49$ & $502.33 \pm 86.32$ \\
\hline Resting membrane potential, mV & $-53.75 \pm 0.87$ & $-53.38 \pm 1.07$ & $-52.45 \pm 1.08$ & $-56.62 \pm 1.40$ & $-53.45 \pm 1.50$ \\
\hline Capacitance, pF & $13.69 \pm 1.21$ & $14.31 \pm 1.68$ & $15.16 \pm 2.32$ & $15.28 \pm 1.99$ & $17.89 \pm 3.52$ \\
\hline Spike rate, $\mathrm{Hz}$ & $21.16 \pm 1.37$ & $26 \pm 2.09$ & $17.75 \pm 1.19^{* * *}$ & $19.93 \pm 1.66$ & $21.22 \pm 3.15$ \\
\hline Rheobase, pA & $73.82 \pm 5.61$ & $70.00 \pm 7.95$ & $77.25 \pm 9.07$ & $74.71 \pm 7.13$ & $73.64 \pm 15.27$ \\
\hline AP threshold, mV & $-39.64 \pm 0.88$ & $-39.01 \pm 1.26$ & $-38.96 \pm 1.11$ & $-40.38 \pm 1.42$ & $-39.49 \pm 1.34$ \\
\hline AP amplitude, mV & $78.41 \pm 1.59$ & $75.54 \pm 2.17$ & $81.06 \pm 2.13$ & $82.06 \pm 2.08$ & $80.90 \pm 2.73$ \\
\hline AP rise, $\mathrm{ms}$ & $6.39 \pm 0.37$ & $6.37 \pm 0.51$ & $6.52 \pm 0.60$ & $6.23 \pm 0.59$ & $5.85 \pm 0.79$ \\
\hline AP half-width, ms & $2.84 \pm 0.12$ & $2.94 \pm 0.22$ & $2.76 \pm 0.16$ & $2.67 \pm 0.13$ & $2.80 \pm 0.25$ \\
\hline AHP amplitude, mV & $-9.34 \pm 0.79$ & $-8.11 \pm 1.00$ & $-10.31 \pm 1.16$ & $-10.03 \pm 1.10$ & $-9.59 \pm 1.49$ \\
\hline AHP rise, ms & $23.79 \pm 1.06$ & $25.83 \pm 1.61$ & $21.91 \pm 1.45$ & $24.83 \pm 1.68$ & $22.04 \pm 1.81$ \\
\hline AHP half-width, ms & $142.91 \pm 10.26$ & $149 \pm 13.53$ & $142.42 \pm 16.87$ & $153.24 \pm 15.71$ & $140.49 \pm 22.91$ \\
\hline
\end{tabular}

All values analyzed at rest $(-50 \mathrm{mV})$. Spike rate at $100 \mathrm{pA}$ step. Data represented as mean \pm SEM. $n=$ number of neurons. Spike rate $\mathrm{P0}-\mathrm{P} 2$ versus $\mathrm{P} 6-\mathrm{P} 7 .{ }^{* * *} p<0.001$.

Table 2. The anatomical position of Dmrt3 neurons in relation to the central canal does not influence their electrical properties

\begin{tabular}{lccc}
\hline & Dorsal & Medial & \multicolumn{1}{c}{ Ventral } \\
\hline$n$ & 16 & 11 & 6 \\
Resting membrane potential, $\mathrm{mV}$ & $-53.11 \pm 1.17$ & $-52.56 \pm 1.54$ & $-53.68 \pm 1.40$ \\
Input resistance, $\mathrm{M} \Omega$ & $509.44 \pm 67.60$ & $342.27 \pm 44.46$ & $377.17 \pm 55.71$ \\
AP amplitude, $\mathrm{mV}$ & $80.48 \pm 2.05$ & $77.04 \pm 2.58$ & $72.45 \pm 6.06$ \\
AP rise, ms & $6.74 \pm 0.51$ & $5.73 \pm 0.88$ & $6.15 \pm 1.23$ \\
AP half-width, ms & $2.87 \pm 0.17$ & $2.99 \pm 0.28$ & $2.87 \pm 0.25$ \\
AHP amplitude, mV & $-10.06 \pm 1.36$ & $-8.05 \pm 1.18$ & $-12.22 \pm 2.56$ \\
AHP rise, ms & $23.46 \pm 1.64$ & $24.49 \pm 2.45$ & $25.10 \pm 4.02$ \\
AHP half-width, ms & $131.72 \pm 14.84$ & $139.49 \pm 20.18$ & $181.39 \pm 25.01$
\end{tabular}

All values analyzed at rest $(-50 \mathrm{mV}) . n=$ number of neurons. Data represented as mean \pm SEM. One-way ANOVA with Bonferroni post-test. $p<0.05$.

Hyperpolarization-activated cation currents $\left(I_{\mathrm{h}}\right)$ are characterized by an inward rectification or "membrane sag" and the occasional production of rebound APs. Inhibitory commissural interneurons within the locomotor circuitry have the hallmark property of a hyperpolarization-activated depolarizing "sag" in response to hyperpolarizing potentials (Wu et al., 2011). A large, inward rectification of the membrane potential was generated in $76 \%$ (20/26) of Dmrt3-Cre neurons from hyperpolarized potentials (inward rectification at $-150 \mathrm{pA}: t_{(18)}=3.197, p=0.005$; at -200 pA: $t_{(12)}=2.42, p=0.04$, unpaired $t$ tests; Figure $\left.3 C, D\right)$, where at the termination of prolonged hyperpolarization, 55\% (11/20) of neurons responded with rebound APs (Fig. 3C). The remaining neurons $(6 / 26)$ had little or no response to the hyperpolarization protocol.

An inward current, indicative of $I_{\mathrm{h}}$, was activated in $62 \%(18 /$ 29) of the Dmrt3-Cre neurons by hyperpolarizing voltage steps ( -60 to $-150 \mathrm{mV}, 1 \mathrm{~s}$ duration, $10 \mathrm{mV}$ increments; Fig. 3-1, available at https://doi.org/10.1523/JNEUROSCI.0326-18.2018. f3-1). The same hyperpolarizing protocol did not activate an inward current in $38 \%(11 / 29)$ of neurons $\left(-140 \mathrm{mV}, I_{\mathrm{h}}\right.$ present vs $I_{\mathrm{h}}$ absent; Mann-Whitney $U=13 n_{(1)}=17, \mathrm{n}_{2}=6, p=0.006$, two-tailed, $-150 \mathrm{mV}, I_{\mathrm{h}}$ present vs $I_{\mathrm{h}}$ absent; Mann-Whitney $U=9 n_{(1)}=16, n_{(2)}=4, p=0.03$, two-tailed; Fig. 3-1, available at https://doi.org/10.1523/JNEUROSCI.0326-18.2018.f3-1). The selective $I_{\mathrm{h}}$ antagonist ZD7288, abolished the depolarizing sag across all current steps and attenuated the inward current confirming that the observed inward current is $I_{\mathrm{h}}$ (Fig. 3-1, available at https://doi.org/10.1523/JNEUROSCI.0326-18.2018.f3-1). $I_{\mathrm{h}}$ does not influence passive or active Dmrt3-Cre properties (Table 1). Recently ZD7288 has been shown to inhibit both $I_{\mathrm{h}}$ and sodium currents (Wu et al., 2012), as such the possible involvement of sodium channels in ZD7288 sensitive currents should be considered.
Dmrt3-Cre neurons are rhythmically active during

\section{fictive locomotion}

To investigate whether Dmrt3 interneurons are active during locomotion, we recorded from $\mathrm{Dmrt} 3^{\mathrm{Cre}}$; $t d \mathrm{~T}$ neurons in a dorsalhorn-removed spinal cord preparation, while monitoring motor output from the ipsilateral ventral roots (VRs; Fig. 4A). Bath application of NMDA, 5-HT, and dopamine induced locomotorlike activity at a frequency of $0.1 \pm 0.05 \mathrm{~Hz}$, and spike activity in 19 of the 20 recorded Dmrt3-Cre neuron-VR pairs. Dmrt3-Cre neurons showed considerable variability in both their burst rhythmicity and firing rate in relation to the VR output during locomotor-like activity (Fig. $4 B$ ). Of the active Dmrt3-VR pairs, $26 \%(5 / 19)$ of Dmrt3-Cre neurons displayed activity bursts that were phase aligned to the ongoing VR activity $(\mathrm{R}>0.5, p<0.05$, Rayleigh test), whereas the remaining neurons $(74 \%, 14 / 19)$ were not aligned with the ongoing VR rhythm. Dmrt3-Cre neurons were recorded both ipsilaterally and contralaterally to the recorded VR rhythm. The activity profiles of all neurons (ipsilateral or contralateral) were then transformed to correlate with the ipsilateral VR rhythm. To identify the preferred phase alignment of Dmrt3-Cre neurons, we extracted the bursting pattern of each neuron by applying a kernel density plot ( $1 \mathrm{~s}$ bins) and plotted in relation to the VR activity bursts (Fig. $4 B, C$ ). This demonstrated a disperse phase preference without any significant tendency for the Dmrt3 population to fire in a given phase of the VR cycle (Fig. 4C). To further determine how the firing of individual Dmrt3-Cre neurons related to the VR activity, von Mises probability densities were calculated for individual spikes across the VR cycles, where the $\kappa$ value represents the degree of coupling between Dmrt3 firing and VR activity. The firing of some Dmrt3-Cre neurons was coupled $(\kappa>0.5, p<$ $0.05)$ to the VR activity $(5 / 19, \kappa=0.89 \pm 0.29$; Fig. $4 D)$, whereas most neurons were uncoupled from the VR activity $(14 / 19, \kappa=0.14 \pm 0.09)$. Dmrt3-Cre neurons exhibited a wide range of firing frequencies $(0.3-5.0 \mathrm{~Hz}$; Fig. $4 E)$ and as a population, Dmrt3-Cre firing was not coupled to the ongoing VR rhythm $(\kappa=0.33 \pm 0.38$; Fig. $4 F)$. Fictive locomotion drugs could potentially influence the electrophysiological properties of Dmrt3-Cre neurons, thus, we recorded a set of cells in the whole cord preparation during drug induced locomotion $(n=$ 7 ) and under non-locomotion conditions $(n=11)$. We found that the input resistance and membrane potential were unchanged in the presence of locomotor drugs (Fig. 4G).

To further characterize Dmrt3-Cre neuronal activity during drug induced locomotor-like activity, we used two-photon $\mathrm{Ca}^{2+}$-imaging using the calcium indicator Oregon Green 
A
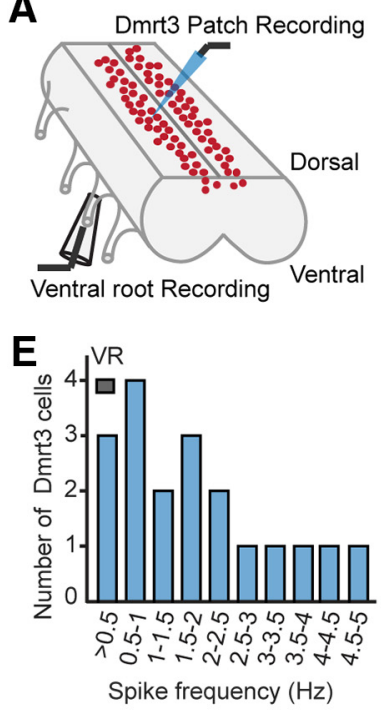

I

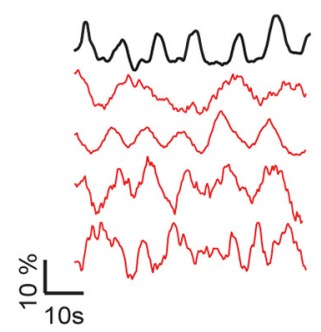

B
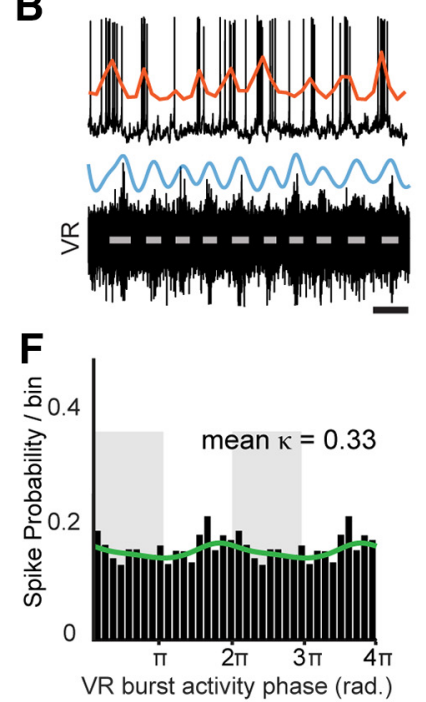

J

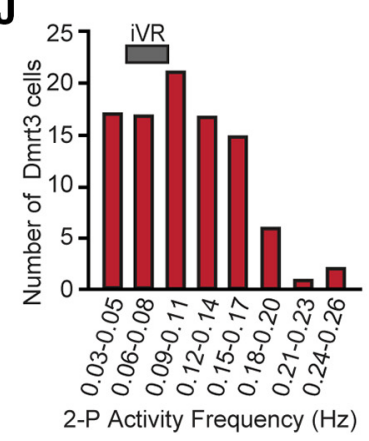

C
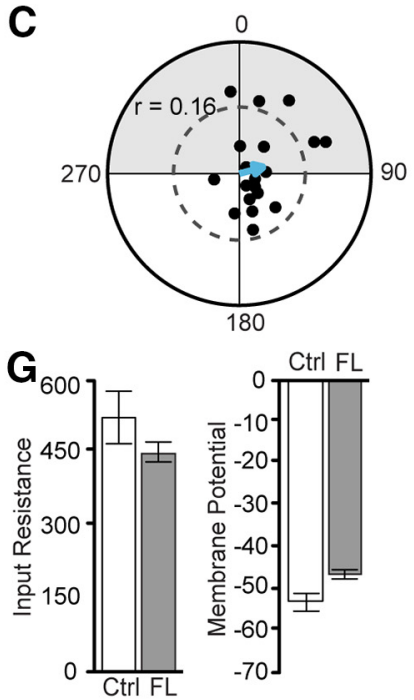

K

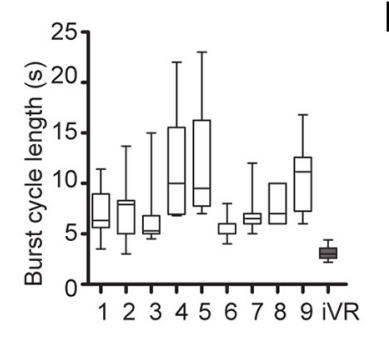

D

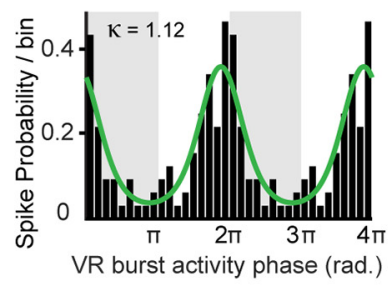

H

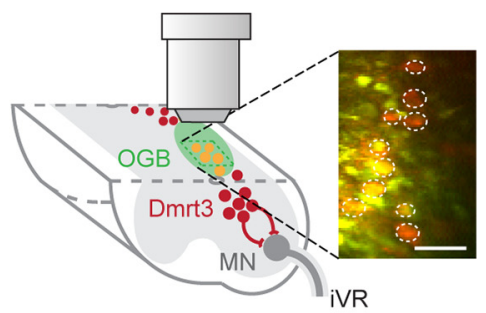

L

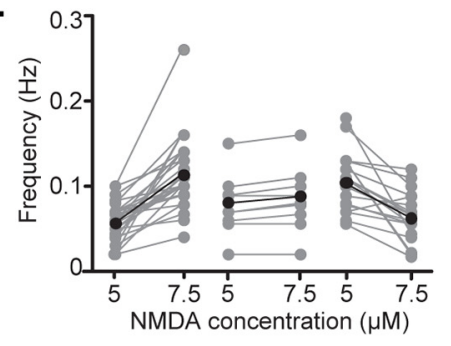

Figure 4. Dmrt3-Cre neurons are active at variable rhythms during fictive locomotion. $A$, Schematic depicting an isolated dorsal-horn-removed spinal cord, patched Dmrt $3{ }^{\text {Cre }}$; $t d T$ neuron and simultaneous recording of ventral root (VR) activity. $\boldsymbol{B}$, Simultaneous recordings from a single Dmrt3 ${ }^{\text {Cre }}$; $t d T$ neuron (top row) and the ongoing VR burst activity (bottom row). VR cycles are depicted in blue, burst duration in gray, and kernel density estimation of spike probability in orange (1 s bins, top row). Scale bar, $10 \mathrm{~s}$. C, Circular phase-diagram indicating the spike phase preference of the $D_{m r t 3}{ }^{\text {(re }} ; t d T$-VR neurons $(n=19$; circles) across 20 consecutive burst cycles in relation to the ipsilateral VR. Blue vector represents the mean phase, and its length indicates the concentration of phase values around the preferred phase (dashed gray line indicates region of significance with $p<0.05$, Rayleigh's test). D, Spike-phase probability histogram of the same Dmrt3 ${ }^{\text {Cre }}$; $t d T$ neuron-ipsilateral VR pair depicted in $\boldsymbol{B}$, indicating the probability of spikes per 20 degree bins across 20 VR cycles, overlaid with von Mises circular normal distribution in green. $\boldsymbol{E}$, Distribution of spike frequencies in the Dmrt ${ }^{\text {(re }}, t d T$ population in response to fictive locomotor drugs. Spinal cord VR frequency shown in gray $(0.1 \pm 0.05 \mathrm{~Hz})$. $\boldsymbol{F}$, Spike-phase probability histogram of all ( $\left.n=19\right)$ Dmrt $3^{\text {(ree }}$; $t d T$ neurons-ipsilateral VR pairs, indicating the probability of spikes per 20 degree bins across 20 VR cycles, overlaid with von Mises circular normal distribution in green. $\mathbf{G}$, Comparison of input resistance and membrane potential by whole-cell patch-clamp recordings of Dmrt3-Cre cells before (ctrl; $n=11)$ and after ( $F L ; n=7$ ) fictive locomotion drugs. $\boldsymbol{H}$, Schematic of a hemisected spinal cord

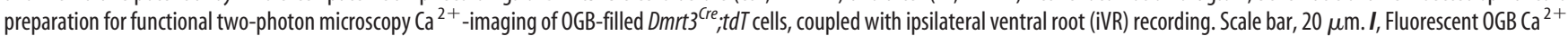
traces $(\Delta F / F)$ showing different oscillatory activities from four Dmrt3 ${ }^{\text {(ree }}$; $t d T$ cells (red) compared with the rectified iVR output (black). J, Distribution of oscillatory frequencies in the Dmrt $3^{\text {Cre }}$; $t d T$ population $(n=97)$ from two-photon microscopy imaging. iVR frequency in gray $(0.07 \mathrm{~Hz}) . \boldsymbol{K}$, Burst cycle length for nine example Dmrt3 ${ }^{\text {(re }}$; $t d T$ cells show high variability compared with the simultaneous iVR rhythm depicted in gray. $L$, Dmrt3 ${ }^{\text {(re }} ;$; tdT oscillation frequencies either increased (left), decreased (right), or remained the same (middle) in response to increased NMDA concentration. Individual Dmrt3 ${ }^{\text {(re }}, t d T$ cells in gray, mean values in black $(n=47)$.

BAPTA-1 AM on midline hemisected or dorsal-horn-shaved spinal cord preparations (P0-P3; Fig. 4H). Hemisected spinal cords showed a markedly slower VR rhythm, as previously described $(0.07 \pm 0.02 \mathrm{~Hz}$; Wilson et al., 2010). Because hemisecting the spinal cord results in the transection of commissural axons, which may alter firing properties, we also used a dorsal-hornshaved preparation (Dougherty and Kiehn, 2010). Measuring activity in both hemisected and dorsal shaved preparations allowed for live $\mathrm{Ca}^{2+}$-imaging of neurons accessed from two tissue surfaces, which should also reduce potential bias. Simultaneously imaged Dmrt3-Cre neurons displayed multiple frequencies in relation to a stable VR rhythm (Fig. 4I). The frequency of Dmrt3Cre neurons showed considerable variability at the individual level throughout recordings, as demonstrated by the large variation in their burst cycle length (Fig. $4 J$ ). This was not dependent on the spinal cord, because individual variability was found in all cords measured. As a population, Dmrt3-Cre neurons $(n=97,5$ spinal cords) could be grouped according to their frequency as either slower $(11 \%, 11 / 97)$, equal to $(20 \%, 19 / 97)$, or faster than $(69 \%, 67 / 97)$ the VR rhythm, emphasizing the multitude of frequencies found among these cells (Fig. $4 K$ ). To investigate the influence of different excitation levels on the Dmrt3-Cre population, we examined their response to higher VR frequency by increasing the NMDA concentration from 5 to $7.5 \mu \mathrm{M}(n=47,2$ cords; Fig. $4 L$ ). We found that while half of the Dmrt3-Cre neurons (23/47 cells, $49 \%$ ) increased their frequency upon increased stimulation, the remaining neurons either decreased $(5 / 47,11 \%)$ or maintained (19/47 cells, 40\%) their frequency. In summary, as a population, Dmrt3-Cre neural firing activity was not correlated to the locomotor rhythm, even though some neurons were found to strongly correlate with motor bursts.

\section{Discussion}

The transcription factor DMRT3 has previously been linked to locomotor function in the mouse and horse (Andersson et al., 2012). Dmrt3 knock-out animals $\left(\right.$ Dmrt $\left.3^{-1-}\right)$ illustrate the re- 
quirement for a functional Dmrt3 gene in the development and generation of coordinated locomotor patterns controlling limb movements (Andersson et al., 2012). In the mouse spinal cord, Dmrt3 defines a subset of inhibitory dI6 interneurons located around the central canal in laminae VII and VIII that are primed to contribute to locomotion. Whereas previous studies have evaluated the properties of putative dI6 neurons (Dyck et al., 2012, Griener et al., 2017) or the Dmrt3 gene (Andersson et al., 2012), this study selectively targets the Dmrt3-derived population. Here we investigated the properties of Dmrt3-Cre spinal cord interneurons, their molecular and electrophysiological character and their activity at the microcircuit level.

Genetically targeted cells that expressed Dmrt3 (Dmrt3 ${ }^{\mathrm{Cre}}$; $t d T$ ) were found throughout the anterior-posterior spinal cord axis, grouped together in lamina VII/VIII. Dmrt3-Cre neurons have commissural, ascending, and descending fibers, receive diverse synaptic inputs, are glycinergic and synapse onto motor neurons. Several rhythmically active CPG interneuron populations have electrophysiological properties, such as spike adaptation and hyperpolarization-activated cation currents $\left(I_{\mathrm{h}}\right)$, which support their inherent rhythmical firing (Kiehn et al., 2000; Butt et al., 2002; Wilson et al., 2005; Dougherty and Kiehn, 2010; Zhong et al., 2010; Borowska et al., 2013; Perry et al., 2015). Dmrt3-Cre neurons have passive membrane properties similar to those described in putative dorsal dI6 cells (Dyck et al., 2012) and display both spike adaptation and $I_{\mathrm{h}}$, which likely contribute to their physiological and functional role. Spike adaptation promotes a neuron's ability to adapt and respond to stimuli (Adibi et al., 2013), whereas $I_{\mathrm{h}}$ contributes to, and is implicated in, rhythmogenesis (Pape, 1996; Kiehn et al., 2000; Leao et al., 2006; He et al., 2014). Thus, the Dmrt3-Cre population are accommodating neurons, of which $60 \%$ display functional $I_{\mathrm{h}}$, a ratio similar to previous reports on the presence of $I_{\mathrm{h}}$ in the V2a population (Zhong et al., 2010).

The presence of $I_{\mathrm{h}}$ indicates that this commissural population is capable of inherent rhythmic firing, and indeed, the activity of Dmrt3-Cre neurons oscillates during fictive locomotion. Both patch-clamp recordings and two-photon $\mathrm{Ca}^{2+}$-based activity imaging revealed that a proportion of Dmrt3-Cre neurons fire rhythmically and quite unexpectedly at diverse frequencies (Fig. 4). To our knowledge, this is the first example of an interneuron population displaying different oscillation frequencies for a given ventral root output rhythm. Previous studies reported that inhibitory CPG interneurons have heterogeneous neuronal firing patterns during locomotor activity (Wu et al., 2011; Dyck et al., 2012). Our findings support these observations, where $63 \%$ of Dmrt3-Cre neurons had rhythmic activity that was either in or out of phase with the recorded ventral root locomotor pattern. The remaining Dmrt3-Cre neurons (47\%) showed no discernible rhythm and fired continuously throughout locomotion in accordance with previous reports (Wu et al., 2011; Dyck et al., 2012). Dmrt3-Cre neurons are likely to be sparsely phase aligned to the ongoing VR rhythm because some neurons, although active, did not show a preferred firing relationship to the ventral root. Moreover, two-photon $\mathrm{Ca}^{2+}$-imaging experiments showed that individual Dmrt3-Cre neurons variably increased or decreased their frequency in response to different excitation levels. Dmrt3 neurons are therefore not likely to be directly involved in generating the CPG rhythm itself. Because the Dmrt3 population simultaneously fires across a range of frequencies, it is possible that they facilitate the shift between locomotor speeds, and such subsets of neurons would then be already "primed" and fire at the required frequencies for continued coordinated output. This is in contrast to previous studies, which reported on rhythmic oscillations of interneurons following the ventral root rhythm (Kwan et al., 2009; Wilson et al., 2010). Such neurons did demonstrate a preferred phase with respect to the CPG output. Indeed, at the population level, Dmrt3 neurons also showed a slight phase preference and fired before peak ventral root activity.

Locomotor interneuron populations, often defined by their gene expression profile, display considerable heterogeneity within the population. For example, during locomotor activity, 53\% of the dI6 neurons were reported to be rhythmically firing during locomotor activity, whereas the remaining $47 \%$ were tonically firing or silent (Dyck et al., 2012). With regard to the dI6 interneurons, it is clear that at least three genetic subpopulations exist, based on the expression of WT1, Dmrt3 or both (Andersson et al., 2012). Evidence suggests that subtypes outside the WT1/Dmrt3-expressing dI6 cells are likely to exist (Griener et al., 2017), but it remains to be established how many subdivisions of the dI6 population exists. Of note, WT1-expressing dI6 neurons also contribute to locomotor circuit function. Recent studies demonstrate that they are inhibitory, their axons are commissural and that they contribute to control of left-right alternation (Haque et al., 2018, Schnerwitzki et al., 2018).

It is yet unclear exactly how Dmrt3 neurons couple to different motor pools or motor modules. Possibly, physiologically distinct active subgroups may work in parallel to maintain and coordinate motor output, where only the relevant Dmrt3 neurons may be recruited for coordination of different gaits. Given that excitatory inputs converge onto Dmrt3-Cre neurons from both sensory (Vglut1) and intrinsic sources (Vglut2), we find it possible that Dmrt3-Cre neurons could modulate excitatory inputs. Together with our previous study (Andersson et al., 2012), Dmrt3-Cre neurons likely participate in locomotor patterning and coordination, and we hypothesize that the capability to oscillate independently of other network inputs could be its defining feature.

We here examined the anatomical, physiological, and functional properties of Dmrt3-Cre neurons in the spinal cord. Dmrt3-Cre neurons project both ipsilaterally and contralaterally, ascending or descending the spinal cord or both. During fictive locomotion, these neurons are active at a wide range of frequencies both with respect to each other and with respect to the ventral root signal. Investigations of spinal Dmrt3-Cre neurons through the attenuation of their activity in freely moving animals are warranted to test the hypotheses advanced here, and to further elucidate the role of Dmrt3-Cre neurons in the control of gaits and motor activation.

\section{References}

Adibi M, McDonald JS, Clifford CW, Arabzadeh E (2013) Adaptation improves neural coding efficiency despite increasing correlations in variability. J Neurosci 33:2108-2120.

Ahituv N, Zhu Y, Visel A, Holt A, Afzal V, Pennacchio LA, Rubin EM (2007) Deletion of ultraconserved elements yields viable mice. PLoS Biol 5:e234.

Alvarez FJ, Villalba RM, Zerda R, Schneider SP (2004) Vesicular glutamate transporters in the spinal cord, with special reference to sensory primary afferent synapses. J Comp Neurol 472:257-280.

Andersson LS, Larhammar M, Memic F, Wootz H, Schwochow D, Rubin CJ, Patra K, Arnason T, Wellbring L, Hjälm G, Imsland F, Petersen JL, McCue ME, Mickelson JR, Cothran G, Ahituv N, Roepstorff L, Mikko S, Vallstedt A, Lindgren G, et al. (2012) Mutations in DMRT3 affect locomotion in horses and spinal circuit function in mice. Nature 488:642-646.

Borowska J, Jones CT, Zhang H, Blacklaws J, Goulding M, Zhang Y (2013) Functional subpopulations of V3 interneurons in the mature mouse spinal cord. J Neurosci 33:18553-18565.

Butt SJ, Harriss-Warrick RM, Kiehn O (2002) Firing properties of identified interneuron populations in the mammalian hindlimb central pattern generator. J Neurosci 22:9961-9971. 
Dougherty KJ, Kiehn O (2010) Functional organization of V2a-related locomotor circuits in the rodent spinal cord. Ann N Y Acad Sci 1198:85-93.

Dumoulin A, Rostaing P, Bedet C, Lévi S, Isambert MF, Henry JP, Triller A, Gasnier B (1999) Presence of the vesicular inhibitory amino acid transporter in GABAergic and glycinergic synaptic terminal boutons. J Cell Sci 112:811-823.

Dyck J, Lanuza GM, Gosgnach S (2012) Functional characterization of dI6 interneurons in the neonatal mouse spinal cord. J Neurophysiol 107:3256-3266

Eide AL, Glover J, Kjaerulff O, Kiehn O (1999) Characterization of commissural interneurons in the lumbar region of the neonatal rat spinal cord. J Comp Neurol 403:332-345.

Enjin A, Rabe N, Nakanishi ST, Vallstedt A, Gezelius H, Memic F, Lind M, Hjalt T, Tourtellotte WG, Bruder C, Eichele G, Whelan PJ, Kullander K (2010) Identification of novel spinal cholinergic genetic subtypes disclose chodl and Pitx2 as markers for fast motor neurons and partition cells. J Comp Neurol 518:2284-2304.

Göbel W, Helmchen F (2007) In vivo calcium imaging of neural network function. Physiology 22:358-365.

Goulding M (2009) Circuits controlling vertebrate locomotion: moving in a new direction. Nat Rev Neurosci 10:507-518.

Griener A, Zhang W, Kao H, Haque F, Gosgnach S (2017) Anatomical and electrophysiological characterization of a population of dI6 interneurons in the neonatal mouse spinal cord. Neuroscience 362:47-59.

Grillner S (1975) Locomotion in vertebrates: central mechanisms and reflex interaction. Physiol Rev 55:247-304.

Haddad GG, Getting PA (1989) Repetitive firing properties of neurons in the ventral region of nucleus tractus solitarius: in vitro studies in adult and neonatal rat. J Neurophysiol 62:1213-1224.

Haque F, Rancic V, Zhang W, Clugston R, Ballanyi K, Gosgnach S (2018) WT1-expressing interneurons regulate left-right alternation during mammalian locomotor activity. J Neurosci 38:5666-5676.

He C, Chen F, Li B, Hu Z (2014) Neurophysiology of HCN channels: from cellular functions to multiple regulations. Prog Neurobiol 112:1-23.

Johannssen HC, Helmchen F (2013) Two-photon imaging of spinal cord cellular networks. Exp Neurol 242:18-26.

Joosten EA, Bär PR, Gispen WH (1995) Collagen implants and corticospinal axonal growth after mid-thoracic spinal cord lesion in the adult rat. J Neurosci Res 41:481-490.

Kiehn O, Kjaerulff O, Tresch MC, Harris-Warrick RM (2000) Contributions of intrinsic motor neuron properties to the production of rhythmic motor output in the mammalian spinal cord. Brain Res Bull 53:649-659.

Kim S, Kettlewell JR, Anderson RC, Bardwell VJ, Zarkower D (2003) Sexually dimorphic expression of multiple doublesex-related genes in the embryonic mouse gonad. Gene Expr Patterns 3:77-82.

Kinnischtzke AK, Sewall AM, Berkepile JM, Fanselow EE (2012) Postnatal maturation of somatostatin-expressing inhibitory cells in the somatosensory cortex of GIN mice. Front Neural Circuits 6:33.

Kjaerulff O, Kiehn O (1996) Distribution of networks generating and coordinating locomotor activity in the neonatal rat spinal cord in vitro: a lesion study. J Neurosci 16:5777-5794.

Kjaerulff O, Kiehn O (1997) Crossed rhythmic synaptic input to motoneurons during selective activation of the contralateral spinal locomotor network. J Neurosci 17:9433-9447.

Kullander K (2005) Genetics moving to neuronal networks. Trends Neurosci 28:239-247.

Kwan AC, Dietz SB, Webb WW, Harris-Warrick RM (2009) Activity of Hb9 interneurons during fictive locomotion in mouse spinal cord. J Neurosci 29:11601-11613.

Lamotte d'Incamps B, Ascher P (2008) Four excitatory postsynaptic ionotropic receptors coactivated at the motoneuron-Renshaw cell synapse. J Neurosci 28:14121-14131.

Langer D, van't Hoff M, Keller AJ, Nagaraja C, Pfäffli OA, Göldi M, Kasper H, Helmchen F (2013) HelioScan: a software framework for controlling in vivo microscopy setups with high hardware flexibility, functional diversity and extendibility. J Neurosci Methods 215:38-52.

Lanuza GM, Gosgnach S, Pierani A, Jessell TM, Goulding M (2004) Genetic identification of spinal interneurons that coordinate left-right locomotor activity necessary for walking movements. Neuron 42:375-386.

Larhammar M, Patra K, Blunder M, Emilsson L, Peuckert C, Arvidsson E, Rönnlund D, Preobraschenski J, Birgner C, Limbach C, Widengren J, Blom H, Jahn R, Wallén-Mackenzie Å, Kullander K (2015) SLC10A4 is a vesicular amine-associated transporter modulating dopamine homeostasis. Biol Psychiatry 77:526-536.

Leao KE, Leao RN, Sun H, Fyffe RE, Walmsley B (2006) Hyperpolarizationactivated currents are differentially expressed in mice brainstem auditory nuclei. J Physiol 576:849-864.

Madisen L, Zwingman TA, Sunkin SM, Oh SW, Zariwala HA, Gu H, Ng LL, Palmiter RD, Hawrylycz MJ, Jones AR, Lein ES, Zeng H (2010) A robust and high-throughput cre reporting and characterization system for the whole mouse brain. Nat Neurosci 13:133-140.

Meyer M, de Angelis MH, Wurst W, Kühn R (2010) Gene targeting by homologous recombination in mouse zygotes mediated by zinc-finger nucleases. Proc Natl Acad Sci U S A 107:15022-15026.

Micallef L, Rodgers P (2014) eulerAPE: drawing area-proportional 3-venn diagrams using ellipses. PloS One 9:e101717.

Mor Y, Lev-Tov A (2007) Analysis of rhythmic patterns produced by spinal neural networks. J Neurophysiol 98:2807-2817.

Pape HC (1996) Queer current and pacemaker: the hyperpolarizationactivated cation current in neurons. Annu Rev Physiol 58:299-327.

Perry S, Gezelius H, Larhammar M, Hilscher MM, Lamotte d'Incamps B, Leao KE, Kullander K (2015) Firing properties of Renshaw cells defined by Chrna 2 are modulated by hyperpolarizing and small conductance ion currents $I_{\mathrm{h}}$ and $I_{\mathrm{SK}}$. Eur J Neurosci 41:889-900.

Rabe N, Gezelius H, Vallstedt A, Memic F, Kullander K (2009) Netrin-1dependent spinal interneuron subtypes are required for the formation of left-right alternating locomotor circuitry. J Neurosci 29:15642-15649.

Schnerwitzki D, Perry S, Ivanova A, Caixeta FV, Cramer P, Günther S, Weber K, Tafreshiha A, Becker L, Vargas Panesso IL, Klopstock T, Hrabe de Angelis M, Schmidt M, Kullander K, and Englert C (2018) Neuronspecific inactivation of Wt1 alters locomotion in mice and changes interneuron composition in the spinal cord. Life Sci Alliance 1:e201800106.

Smith CA, Hurley TM, McClive PJ, Sinclair AH (2002) Restricted expression of DMRT3 in chicken and mouse embryos. Mech Dev 119:S73-76.

Stosiek C, Garaschuk O, Holthoff K, Konnerth A (2003) In vivo two-photon calcium imaging of neuronal networks. Proc Natl Acad Sci U S A 100:7319-7324

Trichas G, Begbie J, Srinivas S (2008) Use of the viral 2A peptide for bicistronic expression in transgenic mice. BMC Biol 6:40.

Vallstedt A, Kullander K (2013) Dorsally derived spinal interneurons in locomotor circuits. Ann N Y Acad Sci 1279:32-42.

Wilson JM, Hartley R, Maxwell DJ, Todd AJ, Lieberam I, Kaltschmidt JA, Yoshida Y, Jessell TM, Brownstone RM (2005) Conditional rhythmicity of ventral spinal interneurons defined by expression of the Hb9 homeodomain protein. J Neurosci 25:5710-5719.

Wilson JM, Blagovechtchenski E, Brownstone RM (2010) Genetically defined inhibitory neurons in the mouse spinal cord dorsal horn: a possible source of rhythmic inhibition of motoneurons during fictive locomotion. J Neurosci 30:1137-1148.

Wu L, Sonner PM, Titus DJ, Wiesner EP, Alvarez FJ, Ziskind-Conhaim L (2011) Properties of a distinct subpopulation of GABAergic commissural interneurons that are part of the locomotor circuitry in the neonatal spinal cord. J Neurosci 31:4821-4833.

Wu X, Liao L, Liu X, Yang T, Li C (2012) Is ZD7288 a selective blocker of hyperpolarization-activated cyclic nucleotide-gated channel currents? Channels (Austin) 6:438-442.

Zagoraiou L, Akay T, Martin JF, Brownstone RM, Jessell TM, Miles GB (2009) A cluster of cholinergic premotor interneurons modulates mouse locomotor activity. Neuron 64:645-662.

Zhong G, Droho S, Crone SA, Dietz S, Kwan AC, Webb WW, Sharma K, Harris-Warrick RM (2010) Electrophysiological characterization of V2a interneurons and their locomotor-related activity in the neonatal mouse spinal cord. J Neurosci 30:170-182. 